REPRESENTATION THEORY

An Electronic Journal of the American Mathematical Society

Volume 11, Pages 16-44 (March 16, 2007)

S 1088-4165(07)00313-5

\title{
CRYSTAL BASES AND SIMPLE MODULES FOR HECKE ALGEBRAS OF TYPE $G(p, p, n)$
}

\author{
JUN HU
}

\begin{abstract}
We apply the crystal basis theory for Fock spaces over quantum affine algebras to the modular representations of the cyclotomic Hecke algebras of type $G(p, p, n)$. This yields a classification of simple modules over these cyclotomic Hecke algebras in the non-separated case, generalizing our previous work [J. Hu, J. Algebra 267 (2003), 7-20]. The separated case was completed in [J. Hu, J. Algebra 274 (2004), 446-490]. Furthermore, we use Naito and Sagaki's work [S. Naito \& D. Sagaki, J. Algebra 251, (2002) 461474] on Lakshmibai-Seshadri paths fixed by diagram automorphisms to derive explicit formulas for the number of simple modules over these Hecke algebras. These formulas generalize earlier results of [M. Geck, Represent. Theory 4 (2000) 370-397] on the Hecke algebras of type $D_{n}$ (i.e., of type $G(2,2, n)$ ).
\end{abstract}

\section{INTRODUCTION}

The theory of crystal (or canonical) bases is one of the most significant advances in Lie theory over the past two decades. It was discovered and developed by M. Kashiwara ([31]) and G. Lusztig ([37]) around 1990. Since then remarkable applications to some classical problems in representation theory have been found. One typical example is the well-known Lascoux-Leclerc-Thibon Conjecture ([35]), which asserts that, the decomposition numbers of the Iwahori-Hecke algebra associated to symmetric group at a primitive $e$-th root of unity in $\mathbb{C}$ (the complex number field) can be obtained from the evaluation at 1 of the coefficient polynomials of natural bases that appeared in the expansion of global crystal bases (i.e., canonical bases) of some level one Fock spaces over the quantum affine algebra $U_{q}\left(\widehat{\mathfrak{s l}}_{e}\right)$. This conjecture has been proved by S. Ariki ([2]), who generalized it to the case of the cyclotomic Hecke algebras of type $G(r, 1, n)$. A similar conjecture ([36]), which relates the decomposition numbers of the $q$-Schur algebra with $q$ specialized to a primitive $e$-th root of unity in $\mathbb{C}$ to global crystal bases of Fock space as $U_{q}\left(\widehat{\mathfrak{g l}}_{e}\right)$-module, has been proved by Varagnolo and Vasserot (43]). For another example, see the work of Brundan and Kleshchev ([10]), where the theory of crystal bases of type $A_{2 l}^{(2)}$ was applied to the modular representations of Hecke-Clifford superalgebras as well as of double covers of symmetric groups.

Received by the editors April 8, 2006.

2000 Mathematics Subject Classification. Primary 20C08, 20C20, 17 B37.

Key words and phrases. Cyclotomic Hecke algebra, Fock space, crystal basis, Kleshchev multipartition, Lakshmibai-Seshadri path.

This research was supported by the National Natural Science Foundation of China (Project 10401005) and by the Program for New Century Excellent Talents in University and partly by the URF of Victoria University of Wellington.

(c) 2007 American Mathematical Society Reverts to public domain 28 years from publication 
This paper provides a new application of the theory of crystal (or canonical) bases to modular representation theory. Precisely, we apply the crystal basis theory for Fock spaces of higher level over the quantum affine algebra of type $A_{l}^{(1)}$ to the modular representations of the cyclotomic Hecke algebra $\mathcal{H}(p, p, n)$ of type $G(p, p, n)$ in the non-separated case (see Definition 3.2). The separated case has been completely solved in our previous work [25]. We explicitly describe (in terms of combinatorics over certain Kleshchev's good lattices) which irreducible representation of the Ariki-Koike algebra $\mathcal{H}(p, n)$ remains irreducible when restricted to $\mathcal{H}(p, p, n)$. This yields a classification of simple modules over the cyclotomic Hecke algebra $\mathcal{H}(p, p, n)$ in the non-separated case, generalizing our previous work 24 on the Hecke algebras of type $D_{n}$. Then we go further in the remaining part of this paper. We use Naito-Sagaki's work ([40, 41]) on Lakshmibai-Seshadri paths fixed by diagram automorphisms to derive an explicit formula for the number of simple modules over the cyclotomic Hecke algebra $\mathcal{H}(p, p, n)$. Our formula generalizes earlier result of Geck [19] on the Hecke algebra of type $D_{n}$ (i.e., of type $G(2,2, n))$. Note that our approach even in that special case is quite different, because it is based on Ariki's celebrated theorem ([2]) on a generalization of the Lascoux-Leclerc-Thibon Conjecture as well as Naito and Sagaki's work (40], 41]) on Lakshmibai-Seshadri paths, while Geck's method in [19 depends on explicit information on character tables and Kazhdan-Lusztig theory for Iwahori-Hecke algebras associated to finite Weyl groups - not presently available in our general $G(p, p, n)$ cases. As a byproduct, we get a remarkable bijection between two sets of Kleshchev multipartitions. Our explicit formulas strongly indicate that there are some new intimate connections between the representation of $\mathcal{H}(p, p, n)$ at roots of unity and the representations of various Ariki-Koike algebras of smaller sizes at various roots of unity. Although we will not discuss these matters in the present paper, we remark that it seems very likely the decomposition matrix of the latter can be naturally embedded as a submatrix of the decomposition matrix of the former.

The paper is organized as follows. Section 2 collects some basic known results about Ariki-Koike algebras (i.e., the cyclotomic Hecke algebras of type $G(r, 1, n)$ ). These include the work of Dipper, James, and Mathas on the structure and representation theory of Ariki-Koike algebras as well as Dipper and Mathas's Morita equivalence results. The notion of Kleshchev multipartition as well as Ariki's remarkable result (Theorem 2.7) are also introduced there. In Section 3, we first recall our previous work on modular representations of Hecke algebras of type $D_{n}$ and of type $G(p, p, n)$. Then we give the first two main results (Theorem 3.6 and Theorem 3.8) in this paper. Theorem 3.9 shows that these two main results are valid over any field $K$ which contains primitive $p$-th root of unity and over which $\mathcal{H}(p, p, n)$ is split. Our Theorem 3.6 is a direct generalization of [24, (1.5)]. A sketch of the proof (following the streamline of the proof of [24, (1.5)]) is presented in Section 4. The proof of Theorem 3.8 is given in Section 5. Our main tools used there are Dipper and Mathas's Morita equivalence results for Ariki-Koike algebras and their connections with type $A$ affine Hecke algebras. In Section 6 we give the second two main results (Theorem 6.10 and Theorem 6.17) in this paper, which yield an explicit formula for the number of simple modules over the cyclotomic Hecke algebra $\mathcal{H}(p, p, n)$ in the non-separated case. Note that in the separated case such a formula can be easily written by using the results [25, (5.7)]. The proof uses our first 
two main results (Theorem 3.6 and Theorem 3.8) as well as the work of Naito and Sagaki (40, 41]) on Lakshmibai-Seshadri paths fixed by diagram automorphisms. As a byproduct, we get (Corollary 6.9) a remarkable bijection between two sets of Kleshchev multipartitions, which seems of independent interest.

The present paper is an expanded version of an earlier preprint (cited in Ariki's book [5, [cyclohecke12]]) completed in the February of 2002. That preprint already contains Theorem 3.6 and Theorem 3.8, which are generalizations of [24, (1.5)]. Part of the remaining work was done during the author's visit at RIMS in 2004. After this expanded version was completed and the main results were announced, N. Jacon informed us that a result similar to Theorem 3.6 in the context of FLOTW partitions (see [29, Definition 2.2]) was also obtained in his Ph.D. Thesis [28] in 2004, and we are informed of the existence of a preprint 20. of Genet and Jacon on the modular representation of the Hecke algebra of type $G(r, p, n)$ (where $p \mid r$ ). Our main results, Theorem 6.10 and Theorem 6.17, are not related to any results in [28] and 20]. Both the paper [20] and the present paper use Ariki's results on Fock spaces, crystal graphs as well as Clifford theory. But [20] uses a different version of Fock space and hence a different parameterization of simple modules over Ariki-Koike algebras. The relationships between the parameterization results given in 20] and our parameterization results given in Theorem 3.6, Theorem 3.8 and in [25, Theorem 4.9] are explained in Remark 3.12.

The author thanks Professor Susumu Ariki for some stimulating discussion, especially for informing him about the work of Naito and Sagaki. The author also thanks Professor Masaki Kashiwara for explaining a result about crystal bases. The main results of this paper were announced at the "International Conference on Representation Theory, III" (Chengdu, August 2004). The author would like to thank Professor Nanhua Xi and Professor Jie Du for their helpful comments. The author also would like to thank the referee for many helpful suggestions.

\section{Preliminaries}

Let $r, p, d$ and $n$ be positive integers such that $p d=r$. The complex reflection group $G(r, p, n)$ is the group consisting of $n$ by $n$ permutation matrices with the properties that the entries are either 0 or $r$-th roots of unity in $\mathbb{C}$, and the $d$-th power of the product of the non-zero entries of each matrix is 1 . The order of $G(r, p, n)$ is $d r^{n-1} n$ !, and $G(r, p, n)$ is a normal subgroup of $G(r, 1, n)$ of index $p$.

Cyclotomic Hecke algebras associated to complex reflection groups were introduced in the work of Broué and Malle ([11]) and in the work of Ariki and Koike ([6]). These algebras are deformations of the group rings of the complex reflection groups. We recall their definitions. Let $K$ be a field and let $q, Q_{1}, \cdots, Q_{r}$ be elements of $K$ with $q$ invertible. Let $\mathcal{H}_{K}(r, n)=\mathcal{H}_{q, Q_{1}, \cdots, Q_{r}}(r, n)$ be the unital $K$-algebra with generators $T_{0}, T_{1}, \cdots, T_{n-1}$ and relations

$$
\begin{aligned}
& \left(T_{0}-Q_{1}\right) \cdots\left(T_{0}-Q_{r}\right)=0, \\
& T_{0} T_{1} T_{0} T_{1}=T_{1} T_{0} T_{1} T_{0}, \\
& \left(T_{i}+1\right)\left(T_{i}-q\right)=0, \quad \text { for } 1 \leq i \leq n-1, \\
& T_{i} T_{i+1} T_{i}=T_{i+1} T_{i} T_{i+1}, \quad \text { for } 1 \leq i \leq n-2, \\
& T_{i} T_{j}=T_{j} T_{i}, \quad \text { for } 0 \leq i<j-1 \leq n-2 .
\end{aligned}
$$


This algebra is called Ariki-Koike algebra or the cyclotomic Hecke algebra of type $G(r, 1, n)$. Whenever the parameter $q$ is clear from the context, we shall say (for simplicity) that the algebra $\mathcal{H}_{K}(r, n)$ is with the parameters set $\left\{Q_{1}, \cdots, Q_{r}\right\}$. This algebra contains the Hecke algebras of type $A$ and type $B$ as special cases. It can be defined over $\mathbb{Z}\left[v, v^{-1}, v_{1}, \cdots, v_{r}\right]$, where $v, v_{1}, \cdots, v_{r}$ are all indeterminates. Upon setting $v=1$ and $v_{i}=(\sqrt[r]{1})^{i-1}$ for each $i$, where $\sqrt[r]{1}$ denotes a primitive $r$-th root of unity in $\mathbb{C}$, one obtains the group algebra for the complex reflection group $G(r, 1, n) \cong \mathbb{Z}_{r} \succ \mathfrak{S}_{n}$.

Suppose that $K$ contains a primitive $p$-th root of unity $\varepsilon$. Let $x_{1}, \cdots, x_{d}$ be invertible elements in $K$ with $x_{i}^{1 / p} \in K$ for each $i$. We consider the Hecke algebra $\mathcal{H}_{K}(r, n)$ with parameters

$$
q, x_{i}^{1 / p} \varepsilon^{j}, \quad i=1,2, \cdots, d, j=0,1, \cdots, p-1 .
$$

Then the first defining relation for $\mathcal{H}_{K}(r, n)$ becomes

$$
\left(T_{0}^{p}-x_{1}\right)\left(T_{0}^{p}-x_{2}\right) \cdots\left(T_{0}^{p}-x_{d}\right)=0 .
$$

Let $\mathcal{H}_{K}(r, p, n)=\mathcal{H}_{q, x_{1}, \cdots, x_{d}}(r, p, n)$ be the subalgebra of $\mathcal{H}_{K}(r, n)$ generated by the elements

$$
T_{0}^{p}, \quad T_{u}:=T_{0}^{-1} T_{1} T_{0}, T_{1}, T_{2}, \cdots, T_{n-1} .
$$

Then it is a $q$-analogue of the group algebra for the complex reflection group $G(r, p, n)$. This algebra is called the cyclotomic Hecke algebra of type $G(r, p, n)$. It is known that in this case (by [38] $) \mathcal{H}_{K}(r, p, n)$ is a symmetric algebra over $K$. For simplicity, we shall often write $\mathcal{H}(r, p, n), \mathcal{H}(r, n)$ instead of $\mathcal{H}_{K}(r, p, n), \mathcal{H}_{K}(r, n)$.

Our main interest in this paper will be the algebra $\mathcal{H}(r, p, n)$ in the special case when $p=r$, that is, the cyclotomic Hecke algebra of type $G(p, p, n)$. The special case $\mathcal{H}(2,2, n)$ is just the Iwahori-Hecke algebra of type $D_{n}$. For convenience, we shall use a normalized version of $\mathcal{H}(p, p, n)$ which is defined as follows. Let $p, n \in \mathbb{N}$. Let $K$ be a field and let $q$ be an invertible element in $K$. Throughout this paper, we assume that $K$ contains primitive $p$-th roots of unity. In particular, char $K$ is coprime to $p$. We define the algebra $\mathcal{H}_{q}(p, n)$ to be the associative unital $K$-algebra with generators $T_{0}, T_{1}, \cdots, T_{n-1}$ subject to the following relations:

$$
\begin{aligned}
& T_{0}^{p}-1=0, \\
& T_{0} T_{1} T_{0} T_{1}=T_{1} T_{0} T_{1} T_{0}, \\
& \left(T_{i}+1\right)\left(T_{i}-q\right)=0, \quad \text { for } 1 \leq i \leq n-1, \\
& T_{i} T_{i+1} T_{i}=T_{i+1} T_{i} T_{i+1}, \quad \text { for } 1 \leq i \leq n-2, \\
& T_{i} T_{j}=T_{j} T_{i}, \quad \text { for } 0 \leq i<j-1 \leq n-2 .
\end{aligned}
$$

Note that if we fix a choice of a primitive $p$-th root of unity $\varepsilon$ in $K$, then the first relation becomes

$$
\left(T_{0}-1\right)\left(T_{0}-\varepsilon\right) \cdots\left(T_{0}-\varepsilon^{p-1}\right)=0,
$$

and the algebra $\mathcal{H}_{q}(p, n)$ is just the Ariki-Koike algebra or the cyclotomic Hecke algebra of type $G(p, 1, n)$ with parameters $q, 1, \varepsilon, \cdots, \varepsilon^{p-1}$. However, the algebra $\mathcal{H}_{q}(p, n)$ itself does not depend on the choice of the primitive $p$-th root of unity. Now, let $\mathcal{H}_{q}(p, p, n)$ be the subalgebra of $\mathcal{H}_{q}(p, n)$ generated by the elements

$$
T_{u}:=T_{0}^{-1} T_{1} T_{0}, T_{1}, T_{2}, \cdots, T_{n-1} .
$$

This algebra, called the cyclotomic Hecke algebra of type $G(p, p, n)$, will be the main subject of this paper. 
It is well-known that $\mathcal{H}_{q}(p, n)$ is a free $\mathcal{H}_{q}(p, p, n)$-module with basis $\left\{1, T_{0}, \cdots\right.$, $\left.T_{0}^{p-1}\right\}$. As a $\mathcal{H}_{q}(p, p, n)$-module, $\mathcal{H}_{q}(p, n)$ is in fact isomorphic to a direct sum of $p$ copies of regular $\mathcal{H}_{q}(p, p, n)$-modules. Let $\tau$ be the $K$-algebra automorphism of $\mathcal{H}_{q}(p, n)$ which is defined on generators by $\tau\left(T_{1}\right)=T_{0}^{-1} T_{1} T_{0}, \tau\left(T_{i}\right)=T_{i}$, for any $i \neq 1$. Let $\sigma$ be the non-trivial $K$-algebra automorphism of $\mathcal{H}_{q}(p, n)$ which is defined on generators by $\sigma\left(T_{0}\right)=\varepsilon T_{0}, \sigma\left(T_{i}\right)=T_{i}$, for any $1 \leq i \leq n-1$. By [25. (1.4)], $\tau\left(\mathcal{H}_{q}(p, p, n)\right)=\mathcal{H}_{q}(p, p, n)$ and clearly $\sigma \downarrow_{\mathcal{H}_{q}(p, p, n)}=$ id. Moreover, the set of $K$-subspaces $\left\{T_{0}^{i} \mathcal{H}_{q}(p, p, n)\right\}_{i=0}^{p-1}$ of $\mathcal{H}_{q}(p, n)$ forms a $\mathbb{Z} / p \mathbb{Z}$-graded Clifford system in $\mathcal{H}_{q}(p, n)$ in the sense of [12, (11.12)].

Our approach to the modular representations of the algebra $\mathcal{H}_{q}(p, p, n)$ is to consider the restriction of the representations of $\mathcal{H}_{q}(p, n)$. To this end, we have to first recall some known results about $\mathcal{H}_{q}(p, n)$. The structure and representation theory for Ariki-Koike algebras with arbitrary parameters $q, Q_{1}, \cdots, Q_{p}$ have been well studied in 14, where it was shown that these algebras are cellular in the sense of [21] if $q$ is invertible. To state their results, we need some combinatorics. Recall that a partition of $n$ is a non-increasing sequence of positive integers $\lambda=$ $\left(\lambda_{1}, \cdots, \lambda_{r}\right)$ such that $|\lambda|:=\sum_{i=1}^{r} \lambda_{i}=n$, while a $p$-multipartition of $n$ is a $p$-tuple of partitions $\lambda=\left(\lambda^{(1)}, \cdots, \lambda^{(p)}\right)$ such that $|\lambda|:=\sum_{i=1}^{p}\left|\lambda^{(i)}\right|=n$. For any two $p$-multipartitions $\lambda$, $\mu$ of $n$, we define $\lambda \unrhd \mu$ if

$$
\sum_{j=1}^{i-1}\left|\lambda^{(j)}\right|+\sum_{k=1}^{m} \lambda_{k}^{(i)} \geq \sum_{j=1}^{i-1}\left|\mu^{(j)}\right|+\sum_{k=1}^{m} \mu_{k}^{(i)}, \quad \forall 1 \leq i \leq p, m \geq 1 .
$$

We shall state the results of $[14$ in our special case of the Ariki-Koike algebra $\mathcal{H}_{q}(p, n)$. First, we fix a primitive $p$-th root of unity $\varepsilon$ in $K$. Let $\mathrm{Q}:=$ $\left\{1, \varepsilon, \varepsilon^{2}, \cdots, \varepsilon^{p-1}\right\}$. Let $\overrightarrow{\mathrm{Q}}=\left(Q_{1}, \cdots, Q_{p}\right)$ be an ordered $p$-tuple which is obtained by fixing an order on $\mathrm{Q}$. We regard $\mathcal{H}_{q}(p, n)$ as the Ariki-Koike algebra with parameters $q, 1, \varepsilon, \cdots, \varepsilon^{p-1}$. Then for each $p$-multipartitions $\lambda$ of $n$, there is a Specht module, denoted by $\widetilde{S}_{\overrightarrow{\mathrm{Q}}}^{\lambda}$, and there is a naturally defined bilinear form $\langle$, on $\widetilde{S}_{\overrightarrow{\mathrm{Q}}}^{\lambda}$. Let $\widetilde{D}_{\overrightarrow{\mathrm{Q}}}^{\lambda}=\widetilde{S}_{\overrightarrow{\mathrm{Q}}}^{\lambda} / \operatorname{rad}\langle$,$\rangle . Note here the subscript \overrightarrow{\mathrm{Q}}$ (instead of Q) is used to emphasize that the structure of the module $\widetilde{S}_{\overrightarrow{\mathrm{Q}}}^{\lambda}$ (resp., $\widetilde{D}_{\overrightarrow{\mathrm{Q}}}^{\lambda}$ ) does depend on the fixed order on the set $\left\{1, \varepsilon, \cdots, \varepsilon^{p-1}\right\}$ of parameters.

Definition 2.1. Let $v$ be an indeterminate over $\mathbb{Z}$. Let $\epsilon$ be a primitive $p$-th root of unity in $\mathbb{C}$. We define $\mathcal{Z}=\mathbb{Z}[\epsilon]\left[v, v^{-1}\right]$ and

$$
f_{p, n}(v, \epsilon)=\prod_{1 \leq i<j \leq p} \prod_{-n<k<n}\left(\epsilon^{i-1} v^{k}-\epsilon^{j-1}\right) \in \mathcal{Z} .
$$

Note that $v^{p}-1=\prod_{k \mid p} \Phi_{k}(v)$, where $\Phi_{k}(v)$ is the $k$-th cyclotomic polynomial over $\mathbb{Z}$. It follows easily that for any $\mathbb{Z}\left[v, v^{-1}\right]$-algebra $K$ which contains a primitive $p$-th root of unity $\varepsilon$, the natural homomorphism $\mathbb{Z}\left[v, v^{-1}\right] \rightarrow K$ can be uniquely extended to a homomorphism from $\mathcal{Z}$ to $K$ by mapping $\epsilon$ to $\varepsilon$. In other words, we can always specialize $\epsilon$ to any primitive $p$-th root of unity.

Theorem 2.2 ([14], [1]). With the above notations, we have that

1) the set $\left\{\widetilde{D}_{\overrightarrow{\mathrm{Q}}}^{\lambda} \mid \lambda\right.$ is a p-multipartition of $n$ and $\left.\widetilde{D}_{\overrightarrow{\mathrm{Q}}}^{\lambda} \neq 0\right\}$ forms a complete set of pairwise non-isomorphic simple $\mathcal{H}_{q}(p, n)$-modules; 
2) if $\widetilde{D}_{\overrightarrow{\mathrm{Q}}}^{\mu} \neq 0$ is a composition factor of $\widetilde{S}_{\overrightarrow{\mathrm{Q}}}^{\lambda}$, then $\lambda \unrhd \mu$, and every composition factor of $\widetilde{S}_{\overrightarrow{\mathrm{Q}}}^{\lambda}$ is isomorphic to some $\widetilde{D}_{\overrightarrow{\mathrm{Q}}}^{\mu}$ with $\lambda \unrhd \mu$; if $\widetilde{D}_{\overrightarrow{\mathrm{Q}}}^{\lambda} \neq 0$, then the composition multiplicity of $\widetilde{D}_{\overrightarrow{\mathrm{Q}}}^{\lambda}$ in $\widetilde{S}_{\overrightarrow{\mathrm{Q}}}^{\lambda}$ is 1 ;

3) $\mathcal{H}_{q}(p, n)$ is semisimple if and only if

$$
\left(\prod_{i=1}^{n}\left(1+q+q^{2}+\cdots+q^{i-1}\right)\right) f_{p, n}(q, \varepsilon) \neq 0
$$

in $K$. In that case, $\widetilde{S}_{\overrightarrow{\mathrm{Q}}}^{\lambda}=\widetilde{D}_{\overrightarrow{\mathrm{Q}}}^{\lambda}$ for each $p$-multipartition $\lambda$ of $n$.

Note that whether $f_{p, n}(q, \varepsilon)$ is non-zero in $K$ or not is independent of the choice of the primitive $p$-th root of unity $\varepsilon$ in $K$.

It remains to determine when $\widetilde{D}_{\overrightarrow{\mathrm{Q}}}^{\lambda} \neq 0$. This was solved in [13] in the case of type $A$. In the general case it was solved by the work of Dipper and Mathas 15] and the work of Ariki [4. We first recall Dipper and Mathas's result in [15]. The two parameters $Q_{i}, Q_{j}$ are said to be in the same $q$-orbit, if $Q_{i}=q^{k} Q_{j}$ for some $k \in \mathbb{Z}$. Now we suppose that $\mathrm{Q}=\mathrm{Q}_{1} \sqcup \mathrm{Q}_{2} \sqcup \cdots \sqcup \mathrm{Q}_{\kappa}$ (disjoint union) such that $Q_{i}, Q_{j}$ are in the same $q$-orbit only if $Q_{i}, Q_{j} \in \mathrm{Q}_{c}$ for some integer $c$ with $1 \leq c \leq \kappa$. Let $p_{i}=\left|\mathrm{Q}_{i}\right|$ for each integer $i$ with $1 \leq i \leq \kappa$.

Theorem 2.3 ([15, Theorem 1.1]). With the above notations, the algebra $\mathcal{H}_{q}(p, n)$ is Morita equivalent to the algebra

$$
\bigoplus_{\substack{n_{1}, \cdots, n_{\kappa} \geq 0 \\ n_{1}+\cdots+n_{\kappa}=n}} \mathcal{H}_{q, Q_{1}}\left(p_{1}, n_{1}\right) \otimes \cdots \otimes \mathcal{H}_{q, Q_{\kappa}}\left(p_{\kappa}, n_{\kappa}\right)
$$

where each $\mathcal{H}_{q, \mathrm{Q}_{i}}\left(p_{i}, n_{i}\right)$ denotes the Ariki-Koike algebra of size $n_{i}$ and with the parameters set $\mathrm{Q}_{i}$. Moreover, if we fix an order on each $\mathrm{Q}_{i}$ to get an ordered tuple $\overrightarrow{\mathrm{Q}}_{i}$ and suppose that $\overrightarrow{\mathrm{Q}}=\left(\overrightarrow{\mathrm{Q}}_{1}, \overrightarrow{\mathrm{Q}}_{2}, \cdots, \overrightarrow{\mathrm{Q}}_{\kappa}\right)$ (concatenation of ordered tuples), then the above Morita equivalence sends $\widetilde{D}_{\overrightarrow{\mathrm{Q}}^{\lambda}}^{\lambda}$ to $\widetilde{D}_{\overrightarrow{\mathrm{Q}}_{1}}^{\lambda^{[1]}} \otimes \cdots \otimes \widetilde{D}_{\overrightarrow{\mathrm{Q}}_{\kappa}}^{\lambda^{[\kappa]}}$, where

$$
\lambda^{[i]}:=\left(\lambda^{\left(\sum_{j=1}^{i-1} p_{j}+1\right)}, \lambda^{\left(\sum_{j=1}^{i-1} p_{j}+2\right)}, \cdots, \lambda^{\left(\sum_{j=1}^{i} p_{j}\right)}\right),\left|\lambda^{[i]}\right|=n_{i}, \forall 1 \leq i \leq \kappa,
$$

$\widetilde{D}_{\overrightarrow{\mathrm{Q}}_{i}}^{\lambda^{[i]}}$ denotes the quotient module (see Definition 2.1 above) of the Specht module $\widetilde{S}_{\overrightarrow{\mathrm{Q}}_{i}}^{\lambda^{[i]}}$ over $\mathcal{H}_{q, \mathrm{Q}_{i}}\left(p_{i}, n_{i}\right)$. In particular, $\widetilde{D}_{\overrightarrow{\mathrm{Q}}}^{\lambda} \neq 0$ if and only if $\widetilde{D}_{\overrightarrow{\mathrm{Q}}_{i}}^{\lambda^{[i]}} \neq 0$ for any integer $i$ with $1 \leq i \leq \kappa$.

Lemma 2.4. Let $\mathcal{H}_{q, \mathrm{Q}}(p, n)$ be the Ariki-Koike algebra with parameters $q, Q_{1}$, $\cdots, Q_{p}$ and defined over $K$. Let $\mathrm{Q}=\left\{Q_{1}, \cdots, Q_{p}\right\}$. Let $0 \neq a \in K$. Let $a \mathrm{Q}=$ $\left\{a Q_{1}, \cdots, a Q_{p}\right\}$. Let $\sigma_{a}$ be the isomorphism from $\mathcal{H}_{q, a \mathrm{Q}}(p, n)$ onto $\mathcal{H}_{q, \mathrm{Q}}(p, n)$ which is defined on generators by $\sigma_{a}\left(T_{0}\right)=a T_{0}$ and $\sigma_{a}\left(T_{i}\right)=T_{i}$ for $i=1,2, \cdots, n-1$. Let $\overrightarrow{\mathrm{Q}}$ be an ordered p-tuple which is obtained by fixing an order on $\mathrm{Q}$. Then for each $p$-multipartition $\lambda$ of $n$, there are $\mathcal{H}_{q, a \mathrm{Q}}(p, n)$-module isomorphisms

$$
\left(\widetilde{S}_{\overrightarrow{\mathrm{Q}}}^{\lambda}\right)^{\sigma_{a}} \cong \widetilde{S}_{\overrightarrow{a \mathrm{Q}}}^{\lambda}, \quad\left(\widetilde{D}_{\overrightarrow{\mathrm{Q}}}^{\lambda}\right)^{\sigma_{a}} \cong \widetilde{D}_{\overrightarrow{a \mathrm{Q}}}^{\lambda}
$$

where $\overrightarrow{\mathrm{aQ}}$ denotes the ordered $p$-tuple which is obtained from $\overrightarrow{\mathrm{Q}}$ by multiplying a on each component. In particular, $\widetilde{D}_{\overrightarrow{\mathrm{Q}}}^{\lambda} \neq 0$ if and only if $\widetilde{D}_{\overrightarrow{a \mathrm{Q}}}^{\lambda} \neq 0$. 
Proof. This follows directly from the definitions and constructions of the modules $\widetilde{S} \underset{\mathrm{Q}}{\lambda}, \widetilde{D}_{\overrightarrow{\mathrm{Q}}}^{\lambda}$.

Theorem 2.3 and Lemma 2.4 reduce the problem of determining when $\widetilde{D}_{\overrightarrow{\mathrm{Q}}}^{\lambda} \neq 0$ to the case where all the parameters in $\mathrm{Q}$ are some powers of $q$. In that case, the problem was solved by Ariki [4. From now on until the end of this section, we assume that all the parameters in $\mathrm{Q}$ are some powers of $q$. In particular, in our $\mathcal{H}_{q}(p, n)$ case, $q$ must be a root of unity. To state the result of Ariki, we have to recall the definition of the Kleshchev multipartition (see [7]). For any $p$-multipartition $\lambda$, the Young diagram of $\lambda$ is the set

$$
[\lambda]=\left\{(a, b, c) \mid 1 \leq c \leq p \text { and } 1 \leq b \leq \lambda_{a}^{(c)}\right\} .
$$

The elements of $[\lambda]$ are nodes of $\lambda$. Given any two nodes $\gamma=(a, b, c), \gamma^{\prime}=\left(a^{\prime}, b^{\prime}, c^{\prime}\right)$ of $\lambda$, say that $\gamma$ is below $\gamma^{\prime}$, or $\gamma^{\prime}$ is above $\gamma$, if either $c>c^{\prime}$ or $c=c^{\prime}$ and $a>a^{\prime}$. The residue of $\gamma=(a, b, c)$ is defined to be

$$
\operatorname{res}(\gamma):=m+e \mathbb{Z} \in \mathbb{Z} / e \mathbb{Z}, \quad \text { if } q=\sqrt[e]{1} \text { and } q^{m}=q^{b-a} Q_{c},
$$

and we say that $\gamma$ is a res $(\gamma)$-node. A removable node is a node of the boundary of $[\lambda]$ which can be removed, while an addable node is a concave corner on the rim of $[\lambda]$ where a node can be added. If $\mu=\left(\mu^{(1)}, \cdots, \mu^{(p)}\right)$ is a $p$-multipartition of $n+1$ with $[\mu]=[\lambda] \cup\{\gamma\}$ for some removable node $\gamma$ of $\mu$, we write $\lambda \rightarrow \mu$. If in addition $\operatorname{res}(\gamma)=x$, we also write that $\lambda \stackrel{x}{\rightarrow} \mu$. For example, suppose $n=10, p=4, q=\sqrt[8]{1}$ and $\varepsilon=q^{2}=\sqrt[4]{1}$. The nodes of $\lambda=\left((2,1),\left(1^{2}\right),\left(1^{3}\right),(2)\right)$ have the following residues

$$
\lambda=\left(\left(\begin{array}{ll}
0 & 1 \\
7 &
\end{array}\right),\left(\begin{array}{l}
2 \\
1
\end{array}\right),\left(\begin{array}{l}
4 \\
3 \\
2
\end{array}\right),\left(\begin{array}{ll}
6 & 7
\end{array}\right)\right) .
$$

It has five removable nodes. Fix a residue $x$ and consider the sequence of removable and addable $x$-nodes obtained by reading the boundary of $\lambda$ from the bottom up. In the above example, we consider residue $x=1$, then we get a sequence "ARR", where each "A" corresponds to an addable $x$-node and each "R" corresponds to a removable $x$-node. Given such a sequence of letters "A,R" , we remove all occurrences of the string "AR" and keep on doing this until no such string "AR" is left. The "R"s that still remain are the normal $x$-nodes of $\lambda$ and the highest of these is the good $x$-node. In the above example, there is only one normal 1-node, which is a good 1-node. If $\gamma$ is a good $x$-node of $\mu$ and $\lambda$ is the multipartition such that $[\mu]=[\lambda] \cup \gamma$, we write $\lambda \stackrel{x}{\rightarrow} \mu$. For each integer $n \geq 0$, let $\mathcal{P}_{n}$ be the set of all $p$-multipartitions of $n$.

Definition 2.6 (7]). Suppose $n \geq 0$. The set $\mathcal{K}_{n}$ of Kleshchev $p$ multipartitions with respect to $\left(q, Q_{1}, \cdots, Q_{p}\right)$ is defined inductively as follows:

(1) $\mathcal{K}_{0}:=\{\underline{\emptyset}:=(\underbrace{\emptyset, \cdots, \emptyset}_{p})\}$

(2) $\mathcal{K}_{n+1}:=\left\{\mu \in \mathcal{P}_{n+1} \mid \lambda \stackrel{x}{\rightarrow} \mu\right.$ for some $\lambda \in \mathcal{K}_{n}$ and some $\left.x\right\}$.

Then Kleshchev's good lattice with respect to $\left(q, Q_{1}, \cdots, Q_{p}\right)$ is, by definition, the infinite graph whose vertices are the Kleshchev $p$-multipartitions with respect 
to $\left(q, Q_{1}, \cdots, Q_{p}\right)$ and whose arrows are given by

$\lambda \stackrel{x}{\rightarrow} \mu \Longleftrightarrow \lambda$ is obtained from $\mu$ by removing a good $x$-node.

Now we can state Ariki's remarkable result (4] ), that is,

Theorem $2.7([4])$. With the above notations, we have that $\widetilde{D}_{\overrightarrow{\mathrm{Q}}}^{\lambda} \neq 0$ if and only if $\lambda$ is a Kleshchev p-multipartition with respect to $\left(q, Q_{1}, \cdots, Q_{p}\right)$.

\section{Classification of Simple $\mathcal{H}_{q}(p, p, n)$-MOdules}

In this section, we shall first review some known results on the classification of simple modules over $\mathcal{H}_{q}(p, p, n)$. Then we shall state the first two main results in this paper, which give a classification of simple modules over $\mathcal{H}_{q}(p, p, n)$ in the non-separated cases. The proof will be given in Section 4 and Section 5.

Recall that $\mathrm{Q}=\left\{1, \varepsilon, \cdots, \varepsilon^{p-1}\right\}$, where $\varepsilon$ is a fixed primitive $p$-th root of unity in $K$. Let $\overrightarrow{\mathrm{Q}}$ be an ordered $p$-tuple which is obtained by fixing an order on $\mathrm{Q}$. Let $K_{n}=\left\{\lambda \in \mathcal{P}_{n} \mid \widetilde{D}_{\overrightarrow{\mathrm{Q}}}^{\lambda} \neq 0\right\}$. The automorphism $\sigma$ determines uniquely an automorphism h of $K_{n}$ such that $\left(\widetilde{D}_{\overrightarrow{\mathrm{Q}}}^{\lambda}\right)^{\sigma} \cong \widetilde{D}_{\overrightarrow{\mathrm{Q}}}^{\mathrm{h}(\lambda)}$. Clearly, $\mathrm{h}^{p}=\mathrm{id}$. In particular, we get an action of the cyclic group $C_{p}$ on $K_{n}$ given as follows: $\widetilde{D}_{\overrightarrow{\mathrm{Q}}}^{\sigma^{k}} \cdot \lambda \cong\left(\widetilde{D}_{\overrightarrow{\mathrm{Q}}}^{\lambda}\right)^{\sigma^{k}}$. Let $\sim_{\sigma}$ be the corresponding equivalence relation on $K_{n}$. That is, $\lambda \sim_{\sigma} \mu$ if and only if $\lambda=g \cdot \mu$ for some $g \in C_{p}$. For each $\lambda \in K_{n} / \sim_{\sigma}$, let $C_{\lambda}$ be the stabilizer of $\lambda$ in $C_{p}$. Then $C_{\lambda}$ is a cyclic subgroup of $C_{p}$ with order $\left|C_{\lambda}\right|$. Clearly $\left|C_{\lambda}\right| \mid p$. We define

$$
K_{n}(0):=\left\{\lambda \in K_{n} / \sim_{\sigma} \mid C_{\lambda}=1\right\}, \quad K_{n}(1):=\left\{\lambda \in K_{n} / \sim_{\sigma} \mid C_{\lambda} \neq 1\right\} .
$$

Lemma $3.1([25,(5.4),(5.5),(5.6)])$. Suppose $\mathcal{H}_{q}(p, p, n)$ is split over $K$.

1) Let $\widetilde{D}_{\overrightarrow{\mathrm{Q}}}^{\lambda}$ be any given irreducible $\mathcal{H}_{q}(p, n)$-module and $D$ be an irreducible $\mathcal{H}_{q}(p, p, n)$-submodule of $\widetilde{D}_{\overrightarrow{\mathrm{Q}}}^{\lambda}$. Let $d$ be the smallest positive integer such that $D \cong$ $D T_{0}^{d}$. Suppose $1 \leq d<p$. Then $k:=p / d$ is the smallest positive integer such that $\widetilde{D}_{\overrightarrow{\mathrm{Q}}}^{\lambda} \cong\left(\widetilde{D}_{\overrightarrow{\mathrm{Q}}}^{\lambda}\right)^{\sigma^{k}}$, and

$$
\widetilde{D}_{\overrightarrow{\mathrm{Q}}}^{\lambda} \downarrow_{\mathcal{H}_{q}(p, p, n)} \cong D \oplus D T_{0} \oplus \cdots \oplus D T_{0}^{d-1}
$$

2) The set

$$
\left\{\widetilde{D}_{\overrightarrow{\mathrm{Q}}}^{\lambda} \downarrow_{\mathcal{H}_{q}(p, p, n)} \mid \lambda \in K_{n}(0)\right\} \bigcup\left\{D^{\lambda, 0}, D^{\lambda, 1}, \cdots, D^{\lambda,\left|C_{\lambda}\right|-1} \mid \lambda \in K_{n}(1)\right\}
$$

forms a complete set of pairwise non-isomorphic simple $\mathcal{H}_{q}(p, p, n)$-modules, where for each $\lambda \in K_{n}(1), D^{\lambda, 0}$ is an irreducible $\mathcal{H}_{q}(p, p, n)$ submodule of $\widetilde{D}_{\overrightarrow{\mathrm{Q}}}^{\lambda}$, and $D^{\lambda, i}=$ $\left(D^{\lambda, 0}\right)^{\tau^{i}}$ for $i=0,1, \cdots,\left|C_{\lambda}\right|-1$.

Therefore, the problem on classifying simple $\mathcal{H}_{q}(p, p, n)$-modules reduces to the problem of determining the automorphism $\mathrm{h}$.

Definition $3.2([3])$. We refer to the condition $f_{p, n}(q, \varepsilon) \in K^{\times}$as the separation condition. We say that we are in the separated case if the separation condition is satisfied, otherwise we are in the non-separated case. 
In the separated case, the classification of all the simple $\mathcal{H}_{q}(p, p, n)$-modules is known by the results in [25]. In particular, we have that

Lemma $3.3([25])$. Suppose $f_{p, n}(q, \varepsilon) \neq 0$ in $K$. Then $\mathcal{H}_{q}(p, p, n)$ is split over $K$, and for any $\lambda=\left(\lambda^{(1)}, \cdots, \lambda^{(p)}\right) \in K_{n}$,

$$
\mathrm{h}(\lambda)=\lambda[1]:=\left(\lambda^{(2)}, \lambda^{(3)}, \cdots, \lambda^{(p)}, \lambda^{(1)}\right) .
$$

The above result generalizes the corresponding results in [42, (3.6),(3.7)] and 23 on the Iwahori-Hecke algebra of type $D_{n}$. So it remains to deal with the case when $f_{p, n}(q, \varepsilon)=0$, i.e., the separated case. In this case, by Theorem 2.7, $K_{n}=\mathcal{K}_{n}$. Henceforth we identify $K_{n}$ with $\mathcal{K}_{n}$ without further comments. Note that in the special case where $p=2, q$ must be a primitive $(2 \ell)$-th root of unity for some positive integer $\ell$ and the classification of all the simple modules is also known by the results in 24]. In that case, $\mathrm{h}$ is an involution. We have that

Lemma 3.4 ([24, (1.4)], [26, Appendix]). Suppose $q$ is a primitive $2 \ell$-th root of unity for some positive integer $\ell$. Suppose char $K \neq 2$ and $\mathcal{H}_{q}\left(D_{n}\right)$ is split over $K$. Let $\lambda \in \mathcal{K}_{n}$ be a Kleshchev bipartition (i.e., 2-multipartition) of $n$ with respect to $(\sqrt[2 e]{1}, 1,-1)$, and let

$$
\underline{\emptyset} \stackrel{r_{1}}{\rightarrow} \cdot \stackrel{r_{2}}{\rightarrow} \ldots \ldots \stackrel{r_{n}}{\rightarrow} \lambda
$$

be a path from $\underline{\emptyset}$ to $\lambda$ in Kleshchev's good lattice with respect to $(\sqrt[2 \ell]{1}, 1,-1)$. Then, the sequence

$$
\underline{\emptyset} \stackrel{\ell+r_{1}}{\rightarrow} \cdot \stackrel{\ell+r_{2}}{\rightarrow} \ldots \ldots \stackrel{\ell+r_{n}}{\rightarrow}
$$

also defines a path in Kleshchev's good lattice with respect to $(\sqrt[2 \ell]{1}, 1,-1)$, and it connects $\underline{\emptyset}$ to $\mathrm{h}(\lambda)$.

Note that the above description of the involution $\mathrm{h}$ bears much resemblance to Kleshchev's description of the well-known Mullineux involution (see 32], 9]).

The first one of the two main results in this section is a direct generalization of Lemma 3.4 to the case of the cyclotomic Hecke algebra $\mathcal{H}_{q}(p, p, n)$. By assumption, $K$ is a field which contains a primitive $p$-th root of unity $\varepsilon$. In particular, char $K$ is coprime to $p$. Now $f_{p, n}(q, \varepsilon)=0$ implies that $\langle\varepsilon\rangle \cap\langle q\rangle \neq\{1\}$, where $\langle\varepsilon\rangle$ (resp., $\langle q\rangle$ ) denotes the multiplicative subgroup generated by $\varepsilon$ (resp., by $q$ ). Let $1 \leq k<p$ be the smallest positive integer such that $\varepsilon^{k} \in\langle q\rangle$. In particular, $k \mid p$. Suppose $\varepsilon^{k}=q^{\ell}$, where $\ell>0$. Let $d=p / k$, then $q^{\ell}=\varepsilon^{k}$ is a primitive $d$-th root of unity. Hence $q$ is a primitive $\left(d \ell_{1}\right)$-th root of unity for some positive integer $\ell_{1}$ with $\ell_{1} \mid \ell$. We need the following result from number theory.

Lemma 3.5. Let $K$ be a field which contains a primitive $p$-th root of unity $\varepsilon$. Suppose $p=d k$, where $p, d, k \in \mathbb{N}$. $\xi \in K$ is a primitive $d$-th root of unity. Then there exists a primitive $p$-th root of unity $\zeta \in K$ such that $\zeta^{k}=\xi$.

Proof. Clearly $\varepsilon^{k}$ is a primitive $d$-th root of unity in $K$. Indeed, the set $\left\{1, \varepsilon, \varepsilon^{2}\right.$, $\left.\cdots, \varepsilon^{p-1}\right\}$ is the set of all $p$-th roots of unity in $K$, and the set $\left\{1, \varepsilon^{k}, \varepsilon^{2 k}, \cdots, \varepsilon^{(d-1) k}\right\}$ is the set of all $d$-th roots of unity in $K$. It follows that there exists some integer $1 \leq a<d$ with $(a, d)=1$ and such that $\xi=\varepsilon^{a k}$.

We write $k=k^{\prime} k^{\prime \prime}$, where $\left(k^{\prime}, d\right)=1$ and any prime factor of $k^{\prime \prime}$ is a factor of $d$. By the Chinese Remainder Theorem, $\mathbb{Z}_{k^{\prime} d} \cong \mathbb{Z}_{k^{\prime}} \times \mathbb{Z}_{d}$. Then we can find $j$ such that $a+j d \equiv 1\left(\bmod k^{\prime}\right)$. In particular, $\left(a+j d, k^{\prime}\right)=1$. It follows that $(a+j d, k)=1$, and hence $(a+j d, p)=1$ (because $(a, d)=1)$. Now $\zeta:=\varepsilon^{a+j d}$ is 
a primitive $p$-th root of unity, and $\zeta^{k}=\varepsilon^{a k+j d k}=\varepsilon^{a k+j p}=\xi$. This completes the proof of the lemma.

We return to our discussion above Lemma 3.5. Note that $q$ is a primitive $\left(d \ell_{1}\right)$-th root of unity implies that $q^{\ell_{1}}$ is a primitive $d$-th root of unity. By Lemma 3.5. we can always find a primitive $p$-th root of unity $\widetilde{\varepsilon}$ such that $(\widetilde{\varepsilon})^{k}=q^{\ell_{1}}$. Since both $\varepsilon$ and $\widetilde{\varepsilon}$ are primitive $p$-th roots of unity, it follows that there exist integers $i, j$, such that $\varepsilon=(\widetilde{\varepsilon})^{i}$ and $\widetilde{\varepsilon}=\varepsilon^{j}$. In particular, $\varepsilon^{m} \in\langle q\rangle$ if and only if $(\widetilde{\varepsilon})^{m} \in\langle q\rangle$ for any integer $m \geq 0$. Therefore, $1 \leq k<p$ is also the smallest positive integer such that $(\widetilde{\varepsilon})^{k} \in\langle q\rangle$. Replacing $\varepsilon$ by $\widetilde{\varepsilon}$ (which makes the Hecke algebra $\mathcal{H}_{q}(p, n$ ) itself unchanged) if necessary, we can assume without loss of generality that $\ell=\ell_{1}$. Henceforth, we fix such $\varepsilon$. Therefore, $q$ is a primitive d $\ell$-th root of unity, $q^{\ell}=\varepsilon^{k}$ is a primitive $d$-th root of unity, and $1 \leq k<p$ is still the smallest positive integer such that $\varepsilon^{k} \in\langle q\rangle$.

For the integer $i=1,2, \cdots, k$, we set $\overrightarrow{\mathrm{Q}}_{i}=\left(\varepsilon^{i-1}, \varepsilon^{k+i-1}, \cdots, \varepsilon^{(d-1) k+i-1}\right)$. Then $\mathrm{Q}=\mathrm{Q}_{1} \sqcup \cdots \sqcup \mathrm{Q}_{k}$ is a partition of the parameter set $\mathrm{Q}$ into different $q$ orbits. Let $\overrightarrow{\mathrm{Q}}=\left(\overrightarrow{\mathrm{Q}}_{1}, \overrightarrow{\mathrm{Q}}_{2}, \cdots, \overrightarrow{\mathrm{Q}}_{k}\right)$ (concatenation of ordered tuples). For each $p$-multipartition $\lambda=\left(\lambda^{(1)}, \cdots, \lambda^{(p)}\right)$ of $n$, we write

$$
\lambda^{[i]}=\left(\lambda^{((i-1) d+1)}, \lambda^{((i-1) d+2)}, \cdots, \lambda^{(i d)}\right), \text { for } i=1,2, \cdots, k \text {. }
$$

and we use $\theta$ to denote the map $\lambda \mapsto\left(\lambda^{[1]}, \cdots, \lambda^{[k]}\right)$. Now we can state the first main result, which deals with the case where $k=1$ and $K=\mathbb{C}$.

Theorem 3.6. Suppose that $K=\mathbb{C}$, and $q, \varepsilon \in K$ such that $\varepsilon=q^{\ell}$ is a primitive $p$-th root of unity and $q$ is a primitive $(p \ell)$-th root of unity. Recall our definition of $\mathrm{h}$ in the second paragraph of this section. Let $\lambda \in \mathcal{K}_{n}$ be a Kleshchev p-multipartition of $n$ with respect to $\left(q, 1, \varepsilon, \cdots, \varepsilon^{p-1}\right)$, and let

$$
\underline{\emptyset} \stackrel{r_{1}}{\rightarrow} \cdot \stackrel{r_{2}}{\rightarrow} \ldots \cdots \stackrel{r_{n}}{\rightarrow} \lambda
$$

be a path from $\underline{\emptyset}$ to $\lambda$ in Kleshchev's good lattice with respect to $\left(q, 1, \varepsilon, \cdots, \varepsilon^{p-1}\right)$. Then, the sequence

$$
\underline{\emptyset} \stackrel{\ell+r_{1}}{\rightarrow} \cdot \stackrel{\ell+r_{2}}{\rightarrow} \cdots \cdots \stackrel{\ell+r_{n}}{\rightarrow}
$$

also defines a path in Kleshchev's good lattice with respect to $\left(q, 1, \varepsilon, \cdots, \varepsilon^{p-1}\right)$, and it connects $\underline{\emptyset}$ to $\mathrm{h}(\lambda)$.

The proof of Theorem 3.6 will be given in Section 4. Here we give an example. Suppose that $n=p=3, q=\sqrt[6]{1}$ and $\varepsilon=q^{2}$. Then the following are all Kleshchev 3 -multipartitions with respect to $\left(q, 1, \varepsilon, \varepsilon^{2}\right)$ of 3 :

$$
\begin{aligned}
& \left(\emptyset, \emptyset,\left(1^{3}\right)\right),(\emptyset, \emptyset,(2,1)),(\emptyset, \emptyset,(3)),\left(\emptyset,(1),\left(1^{2}\right)\right),(\emptyset,(1),(2)), \\
& \left(\emptyset,\left(1^{2}\right),(1)\right),\left(\emptyset,\left(1^{3}\right), \emptyset\right),(\emptyset,(2),(1)),(\emptyset,(2,1), \emptyset),\left((1), \emptyset,\left(1^{2}\right)\right), \\
& ((1), \emptyset,(2)),((1),(1),(1)),\left((1),\left(1^{2}\right), \emptyset\right),((1),(2), \emptyset), \quad\left(\left(1^{2}\right), \emptyset,(1)\right), \\
& \left(\left(1^{2}\right),(1), \emptyset\right), \quad((2), \emptyset,(1)), \quad((2),(1), \emptyset), \quad((2,1), \emptyset, \emptyset),
\end{aligned}
$$


and the automorphism $\mathrm{h}$ is given by

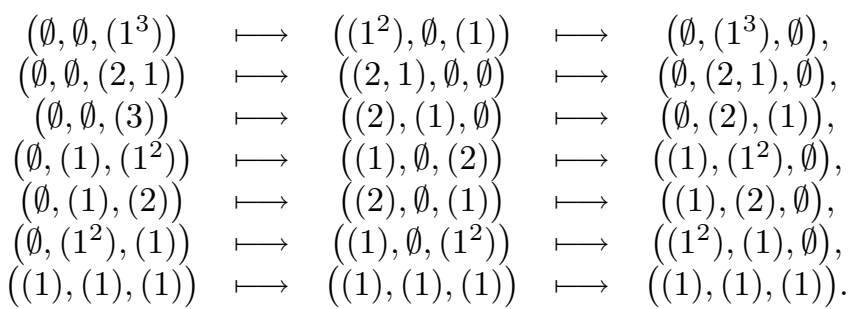

In view of Theorem 3.6 and the discussion above it, it remains to consider the case where $k>1$. Now we suppose $k>1$. Recall our assumption, that is, $q$ is a primitive $(d \ell)$-th root of unity, $q^{\ell}=\varepsilon^{k}$ is a primitive $d$-th root of unity, and $1 \leq k<p$ is the smallest positive integer such that $\varepsilon^{k} \in\langle q\rangle$. Let $\varepsilon^{\prime}=\varepsilon^{k}$, which is a primitive $d$-th root of unity in $\mathbb{C}$. For any integer $n^{\prime} \geq 1$, let $\mathcal{H}_{q}\left(d, n^{\prime}\right)$ be the Ariki-Koike algebra (over $\mathbb{C}$ ) with parameters $\left\{q, 1, \varepsilon^{\prime},\left(\varepsilon^{\prime}\right)^{2}, \cdots,\left(\varepsilon^{\prime}\right)^{d-1}\right\}$ and size $n^{\prime}$. Let $\sigma^{\prime}$ be the non-trivial $\mathbb{C}$-algebra automorphism of $\mathcal{H}_{q}\left(d, n^{\prime}\right)$ which is defined on generators by $\sigma\left(T_{0}\right)=\varepsilon^{\prime} T_{0}, \sigma\left(T_{i}\right)=T_{i}$, for $i=1,2, \cdots, n^{\prime}-1$. Let $\mathcal{K}_{n^{\prime}}^{\prime}$ be the set of Kleshchev $d$-multipartitions of $n^{\prime}$ with respect to $\left(q, 1, \varepsilon^{\prime},\left(\varepsilon^{\prime}\right)^{2}, \cdots,\left(\varepsilon^{\prime}\right)^{d-1}\right)$. Let $\overrightarrow{\mathrm{Q}}^{\prime}:=\left(1, \varepsilon^{\prime},\left(\varepsilon^{\prime}\right)^{2}, \cdots,\left(\varepsilon^{\prime}\right)^{d-1}\right)$. Note that all the parameters in $\overrightarrow{\mathrm{Q}}^{\prime}$ are in a single $q$-orbit. For any $d$-multipartition $\lambda^{\prime}$ of $n^{\prime}$, by Theorem $2.7 \widetilde{D} \widetilde{\vec{Q}}_{\overrightarrow{Q^{\prime}}}^{\lambda^{\prime}} \neq 0$ if and only if $\lambda^{\prime} \in \mathcal{K}_{n^{\prime}}^{\prime}$. The automorphism $\sigma^{\prime}$ determines uniquely an automorphism $\mathrm{h}^{\prime}$ of $\mathcal{K}_{n^{\prime}}^{\prime}$ such that $\left(\widetilde{D}_{\overrightarrow{\mathrm{Q}}^{\prime}}^{\lambda^{\prime}}\right)^{\sigma^{\prime}} \cong \widetilde{D}_{\overrightarrow{\mathrm{Q}}^{\prime}}^{\mathrm{h}^{\prime}\left(\lambda^{\prime}\right)}$. Clearly, $\left(\mathrm{h}^{\prime}\right)^{d}=\mathrm{id}$. Note that $\varepsilon^{\prime}=\varepsilon^{k}=q^{\ell}$. Hence we are in a position to apply Theorem 3.6 with $p$ replaced by $d, n$ replaced by $n^{\prime}$ and $\varepsilon$ replaced by $\varepsilon^{\prime}$. We get the following.

Corollary 3.7. Let $\lambda^{\prime} \in \mathcal{K}_{n^{\prime}}^{\prime}$ be a Kleshchev d-multipartition of $n^{\prime}$ with respect to $\left(q, 1, \varepsilon^{\prime}, \cdots,\left(\varepsilon^{\prime}\right)^{d-1}\right)$, and let

$$
(\underbrace{\emptyset, \cdots, \emptyset}_{d})=\underline{\emptyset} \stackrel{r_{1}}{\rightarrow} \cdot \stackrel{r_{2}}{\rightarrow} \ldots \ldots \stackrel{r_{n^{\prime}}}{\rightarrow} \lambda^{\prime}
$$

be a path from $\underline{\emptyset}$ to $\lambda^{\prime}$ in Kleshchev's good lattice with respect to $\left(q, 1, \varepsilon^{\prime}, \cdots\right.$, $\left.\left(\varepsilon^{\prime}\right)^{d-1}\right)$. Then, the sequence

$$
(\underbrace{\emptyset, \cdots, \emptyset}_{d})=\underline{\emptyset} \stackrel{\ell+r_{1}}{\rightarrow} \cdot \stackrel{\ell+r_{2}}{\rightarrow} \ldots \ldots \stackrel{\ell+r_{n^{\prime}}}{\rightarrow} .
$$

also defines a path in Kleshchev's good lattice with respect to $\left(q, 1, \varepsilon^{\prime}, \cdots,\left(\varepsilon^{\prime}\right)^{d-1}\right)$, and it connects $\underline{\emptyset}$ to $\mathrm{h}^{\prime}\left(\lambda^{\prime}\right)$.

Now we can state the second main result, which deals with the case where $k>1$ and $K=\mathbb{C}$.

Theorem 3.8. Suppose that $K=\mathbb{C}, q, \varepsilon \in K$ such that $\varepsilon$ is a primitive $p$-th

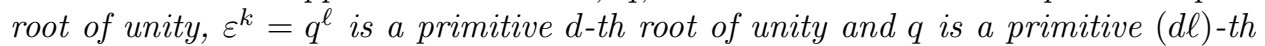
root of unity, and $1 \leq k<p$ is the smallest positive integer such that $\varepsilon^{k} \in\langle q\rangle$. Let $\lambda \in \mathcal{K}_{n}$ be a Kleshchev p-multipartition of $n$ with respect to $(q, \overrightarrow{\mathrm{Q}})$, where $\overrightarrow{\mathrm{Q}}=\left(\overrightarrow{\mathrm{Q}}_{1}, \overrightarrow{\mathrm{Q}}_{2}, \cdots, \overrightarrow{\mathrm{Q}}_{k}\right)$ (concatenation of ordered tuples). Then

$$
\theta(\mathrm{h}(\lambda))=\left(\mathrm{h}^{\prime}\left(\lambda^{[k]}\right), \lambda^{[1]}, \cdots, \lambda^{[k-1]}\right)
$$


where $\left|\lambda^{[k]}\right|=n^{\prime}, \mathrm{h}^{\prime}$ is as defined in Corollary 3.7 and the right-hand side of the above equality is understood as concatenation of ordered tuples.

The proof of Theorem 3.8 will be given in Section 5 .

Theorem 3.9. Both Theorem 3.6 and Theorem 3.8 remain true if we replace $\mathbb{C}$ by any field $K$ such that $\mathcal{H}_{q}(p, p, n)$ is split over $K$ and $K$ contains a primitive $p$-th root of unity.

Proof. In the case where $p=2$, this is proved in the appendix of 26] (which is essentially an argument due to S. Ariki). In general, this can still be proved by using the same argument as in the appendix of [26].

Note that we have proved in [25. Theorem 5.7] that in the separated case $\mathcal{H}_{q}(p, p, n)$ is always split over $K$ whenever $K$ contains a primitive $p$-th root of unity. It would be interesting to know if this is still true in the non-separated case.

Remark 3.10. Let $\overrightarrow{\mathrm{Q}}:=\left(Q_{1}, \cdots, Q_{p}\right)$ be an arbitrary permutation of $\left(1, \varepsilon, \cdots, \varepsilon^{p-1}\right)$. We redefine the residue of a node $\gamma=(a, b, c)$ to be $\operatorname{res}(\gamma):=q^{b-a} Q_{c}$. The notions of good nodes and Kleshchev multipartitions are defined in a similar way as before. For any $r \in K$, we use $\lambda \stackrel{r}{\rightarrow} \mu$ to indicate $\lambda$ is obtained from $\mu$ by removing a good $r$-node. One can easily deduce from Theorem 3.6 and Theorem 3.8 the following description of h: Let $\lambda \in \mathcal{K}_{n}$ be a Kleshchev $p$-multipartition of $n$ with respect to $\left(q, Q_{1}, \cdots, Q_{p}\right)$, and let $\underline{\emptyset} \stackrel{r_{1}}{\rightarrow} \cdot \stackrel{r_{2}}{\rightarrow} \cdots \cdots \stackrel{r_{n}}{\rightarrow} \lambda$ be a path from $\underline{\emptyset}$ to $\lambda$ in Kleshchev's good lattice with respect to $\left(q, Q_{1}, \cdots, Q_{p}\right)$. Then, the sequence

$$
\underline{\emptyset} \stackrel{\varepsilon r_{1}}{\rightarrow} \stackrel{\varepsilon r_{2}}{\rightarrow} \ldots \cdots \stackrel{\varepsilon r_{n}}{\rightarrow}
$$

also defines a path in Kleshchev's good lattice with respect to $\left(q, Q_{1}, \cdots, Q_{p}\right)$, and it connects $\underline{\emptyset}$ to $\mathrm{h}(\lambda)$.

Remark 3.12. In the case of $G(r, p, n)$, one can still define the automorphisms $\sigma, \tau$ and $\mathrm{h}$, and the problem of classifying simple $\mathcal{H}_{q}(r, p, n)$-modules can still be reduced to the determination of $\mathrm{h}$. Note that although we deal with the $G(p, p, n)$ case in this paper, it is not difficult to generalize Theorem 3.6 and Theorem 3.8 in this paper to the $G(r, p, n)$ case by using the same argument as well as the Morita equivalence theorem ([15, Theorem 1.1]) of Dipper and Mathas for Ariki-Koike algebra:1. In the preprint [20], Genet and Jacon give a parameterization of simple $\mathcal{H}_{q}(r, p, n)$ modules when $q$ is a root of unity by using combinatorics of FLOTW partitions. Our $\sigma, \tau$, h are denoted by $f, g, \tau$ in their paper. They give a characterization of $\tau$ in terms of $\omega$ and a bijection $\kappa$ between the set of Kleshchev multipartitions and the set of FLOTW partitions (see [20, Proposition 2.9, lines 3-4 on page 14]). Note that our description of $\mathrm{h}$ in (3.11) is actually a statement about the crystal graph, and although [20] uses JMMO's Fock space ([30, [16]) and we use Hayashi's Fock space $([22,[7])$, the two crystals provided by the lattice of Kleshchev multipartitions and by the lattice of FLOTW partitions respectively are isomorphic to each other. It follows that the description of $h$ in (3.11) in the context of Kleshchev's good lattices should also be valid in the context of FLOTW's good lattices. Therefore, there is a second approach which can be used to generalize Theorem 3.6 and Theorem 3.8

\footnotetext{
${ }^{1}$ We do not deal with the general $G(r, p, n)$ case here because this paper has already been cited in Ariki's book [5. [cyclohecke12]] on the one hand; on the other hand, the proof in the $G(r, p, n)$ case needs nothing more than the proof in the $G(p, p, n)$ case except some sophisticated notations.
} 
in this paper to the $G(r, p, n)$ case. The details are given in [27. The main idea is to derive from the setting of FLOTW partitions and 20, Proposition 2.9, lines $3-4$ on page 14]) a description of $h$ like (3.11) (see [28, (4.3.1)] for the special case when $p=r$ and $\varepsilon=q^{\ell}$ ). In the case where $p=r$, the parameterization of simple modules obtained in [20] is the same as [25, Theorem 5.7] in the separated case. It is worthwhile and interesting to establish a direct connection between the two parameterizations of irreducible representations.

\section{Proof of Theorem 3.6}

In this section, we shall give the proof of Theorem 3.6. It turns out that the proof of Theorem 3.6 is a direct generalization of the proof of [24, (1.5)]. Throughout this section, we keep the same assumptions and notations as in Theorem 3.6. That is, $K=\mathbb{C}, q, \varepsilon \in \mathbb{C}$ be such that $\varepsilon=q^{\ell}$ is a primitive $p$-th root of unity and $q$ is a primitive $(p \ell)$-th root of unity.

Let $v$ be an indeterminate over $\mathbb{Q}$. Let $\mathfrak{h}$ be a $(p \ell+1)$-dimensional vector space over $\mathbb{Q}$ with basis $\left\{h_{0}, h_{1}, \cdots, h_{p \ell-1}, d\right\} 2$. Denote by $\left\{\Lambda_{0}, \Lambda_{1}, \cdots, \Lambda_{p \ell-1}, \delta\right\}$ the corresponding dual basis of $\mathfrak{h}^{*}$, and we set $\alpha_{i}=2 \Lambda_{i}-\Lambda_{i-1}-\Lambda_{i+1}+\delta_{i, 0} \delta$ for $i \in \mathbb{Z} / p \ell \mathbb{Z}$. The weight lattice is $P=\mathbb{Z} \Lambda_{0} \oplus \cdots \oplus \mathbb{Z} \Lambda_{p \ell-1} \oplus \mathbb{Z} \delta$, its dual is $P^{\vee}=$ $\mathbb{Z} h_{0} \oplus \cdots \oplus \mathbb{Z} h_{p \ell-1} \oplus \mathbb{Z} d$. Assume that the $p \ell \times p \ell$ matrix $\left(\left\langle\alpha_{i}, h_{j}\right\rangle\right)$ is just the generalized Cartan matrix associated to $\widehat{\mathfrak{s l}}_{p \ell}$. The quantum affine algebra $U_{v}\left(\widehat{\mathfrak{s l}}_{p \ell}\right)$ is by definition the $\mathbb{Q}(v)$-algebra with 1 generated by elements $E_{i}, F_{i}, i \in \mathbb{Z} / p \ell \mathbb{Z}$ and $K_{h}, h \in P^{\vee}$, subject to the relations

$$
\begin{gathered}
K_{h} K_{h^{\prime}}=K_{h+h^{\prime}}=K_{h^{\prime}} K_{h}, K_{0}=1, \\
K_{h} E_{j}=v^{\left\langle\alpha_{j}, h\right\rangle} E_{j} K_{h}, \quad K_{h} F_{j}=v^{-\left\langle\alpha_{j}, h\right\rangle} F_{j} K_{h}, \\
E_{i} F_{j}-F_{j} E_{i}=\delta_{i j} \frac{K_{h_{i}}-K_{-h_{i}}}{v-v^{-1}}, \\
\sum_{k=0}^{1-\left\langle\alpha_{i}, h_{j}\right\rangle}(-1)^{k}\left[\begin{array}{c}
1-\left\langle\alpha_{i}, h_{j}\right\rangle \\
k
\end{array}\right] E_{i}^{1-\left\langle\alpha_{i}, h_{j}\right\rangle-k} E_{j} E_{i}^{k}=0, \quad(i \neq j), \\
\sum_{k=0}^{1-\left\langle\alpha_{i}, h_{j}\right\rangle}(-1)^{k}\left[\begin{array}{c}
1-\left\langle\alpha_{i}, h_{j}\right\rangle \\
k
\end{array}\right] F_{i}^{1-\left\langle\alpha_{i}, h_{j}\right\rangle-k} F_{j} F_{i}^{k}=0, \quad(i \neq j),
\end{gathered}
$$

where

$$
[k]:=\frac{v^{k}-v^{-k}}{v-v^{-1}}, \quad[k] !:=[k][k-1] \cdots[1], \quad\left[\begin{array}{c}
m \\
k
\end{array}\right]:=\frac{[m] !}{[m-k] ![k] !} .
$$

It is a Hopf algebra with comultiplication given by

$$
\Delta\left(K_{h}\right)=K_{h} \otimes K_{h}, \Delta\left(E_{i}\right)=E_{i} \otimes 1+K_{-h_{i}} \otimes E_{i}, \Delta\left(F_{i}\right)=F_{i} \otimes K_{h_{i}}+1 \otimes F_{i} .
$$

Let $\mathcal{P}^{(1)}$ be the set of all partitions. Let $\mathcal{P}:=\bigsqcup_{n \geq 0} \mathcal{P}_{n}$, the set of all $p$ multipartitions. For each integer $j$ with $0 \leq j<p \ell$, there is a level 1 Fock space $\mathcal{F}^{(1)}\left(\Lambda_{j}\right)$, which is defined as follows. As a vector space,

$$
\mathcal{F}^{(1)}\left(\Lambda_{j}\right):=\bigoplus_{\lambda \in \mathcal{P}^{(1)}} \mathbb{Q}(v) \lambda
$$

\footnotetext{
${ }^{2}$ The readers should not confuse the element $d$ here with the integer $d$ we used before.
} 
and the algebra $U_{v}\left(\widehat{\mathfrak{s l}}_{p \ell}\right)$ acts on $\mathcal{F}^{(1)}\left(\Lambda_{j}\right)$ by

$$
\begin{gathered}
K_{h_{i}} \lambda=v^{N_{i}(\lambda)} \lambda, \quad K_{d} \lambda=v^{-N_{d}(\lambda)} \lambda, \\
E_{i} \lambda=\sum_{\nu \stackrel{i}{\rightarrow} \lambda} v^{-N_{i}^{r}(\nu, \lambda)} \nu, \quad F_{i} \lambda=\sum_{\lambda \stackrel{i}{\rightarrow} \mu} v^{N_{i}^{l}(\lambda, \mu)} \mu,
\end{gathered}
$$

where $i \in \mathbb{Z} / p \ell \mathbb{Z}, \lambda \in \mathcal{P}^{(1)}$, and

$$
\begin{gathered}
N_{i}(\lambda)=\#\{\mu \mid \lambda \stackrel{i}{\rightarrow} \mu\}-\#\{\nu \mid \nu \stackrel{i}{\rightarrow} \lambda\}, \\
N_{i}^{r}(\nu, \lambda)=\sum_{\gamma \in[\lambda] \backslash[\nu]}\left(\#\left\{\gamma^{\prime} \mid \begin{array}{c}
\gamma^{\prime} \text { an addable } \\
i \text {-node of } \lambda \text { above } \gamma
\end{array}\right\}\right. \\
\left.-\#\left\{\gamma^{\prime} \mid \begin{array}{c}
\gamma^{\prime} \text { a removable } \\
i \text {-node of } \nu \text { above } \gamma
\end{array}\right\}\right), \\
N_{i}^{l}(\lambda, \mu)=\sum_{\gamma \in[\mu] \backslash[\lambda]}\left(\#\left\{\gamma^{\prime} \mid \begin{array}{c}
\gamma^{\prime} \text { an addable } \\
i \text {-node of } \mu \text { below } \gamma
\end{array}\right\}\right. \\
\left.-\#\left\{\gamma^{\prime} \mid \begin{array}{c}
\gamma^{\prime} \text { a removable } \\
i \text {-node of } \lambda \text { below } \gamma
\end{array}\right\}\right),
\end{gathered}
$$

and $N_{d}(\lambda):=\#\{\gamma \in[\lambda] \mid \operatorname{res}(\gamma)=0\}$, and here we should use the following definition of residue, namely, the node in the ath row and the $b$ th column of $\lambda$ is filled out with the residue $b-a+j \in \mathbb{Z} / p \ell \mathbb{Z}$.

Let $\Lambda:=\Lambda_{0}+\Lambda_{\ell}+\Lambda_{2 \ell}+\cdots+\Lambda_{(p-1) \ell}$. Replacing $\mathcal{P}^{(1)}$ by $\mathcal{P}$, and using the definition of residue given in (2.5) for multipartition, we get a level $p$ Fock space

$$
\mathcal{F}(\Lambda):=\bigoplus_{\lambda \in \mathcal{P}} \mathbb{Q}(v) \lambda
$$

As a vector space, we have $\mathcal{F}(\Lambda) \cong \mathcal{F}^{(1)}\left(\Lambda_{0}\right) \otimes \cdots \otimes \mathcal{F}^{(1)}\left(\Lambda_{(p-1) \ell}\right), \lambda \mapsto \lambda^{(1)} \otimes \cdots \otimes$ $\lambda^{(p)}$. By [7, (2.5)], it is indeed an $U_{v}\left(\widehat{\mathfrak{s l}}_{p \ell}\right)$-module isomorphism, where the action on the right-hand side is defined via $\Delta^{(p-1)}:=\underbrace{(\Delta \otimes 1 \otimes \cdots \otimes 1)}_{p-1} \cdots \underbrace{(\Delta \otimes 1)}_{2} \underbrace{\Delta}_{1}$.

For each $\lambda=\left(\lambda^{(1)}, \cdots, \lambda^{(p)}\right) \in \mathcal{P}$, we define $\widehat{\lambda}=\left(\lambda^{(p)}, \lambda^{(1)}, \cdots, \lambda^{(p-1)}\right)$. Let $\Theta$ be the automorphism of $\left(U_{v}\left(\widehat{\mathfrak{s l}}_{p \ell}\right)\right)^{\otimes p}$ which is defined by $\Theta\left(x_{1} \otimes \cdots \otimes x_{p}\right)=$ $x_{2} \otimes \cdots \otimes x_{p} \otimes x_{1}$ for any $x_{1}, \cdots, x_{p} \in U_{v}\left(\widehat{\mathfrak{s l}}_{p \ell}\right)$. We now introduce a different version of a level $p$ Fock space $\widehat{\mathcal{F}}(\Lambda)$. As a vector space, $\widehat{\mathcal{F}}(\Lambda)=\mathcal{F}(\Lambda) \cong \mathcal{F}^{(1)}\left(\Lambda_{0}\right) \otimes \cdots \otimes$ $\mathcal{F}^{(1)}\left(\Lambda_{(p-1) \ell}\right)$, while the action (denoted by "०") of $U_{v}\left(\widehat{\mathfrak{s l}}_{p \ell}\right)$ is defined by

$$
x \circ\left(\lambda^{(1)} \otimes \cdots \otimes \lambda^{(p)}\right):=\left\{\Theta\left(\Delta^{(p-1)}(x)\right)\right\}\left(\lambda^{(1)} \otimes \cdots \otimes \lambda^{(p)}\right) .
$$

Lemma 4.1. The above action defines an integrable representation of the algebra $U_{v}\left(\widehat{\mathfrak{s l}}_{p \ell}\right)$ on $\widehat{\mathcal{F}}(\Lambda)$, such that

$$
\begin{aligned}
& K_{h_{i}} \circ \lambda=v^{N_{i}(\lambda)} \lambda, \quad K_{d} \circ \lambda=v^{-N_{d}(\lambda)} \lambda, \\
& E_{i} \circ \lambda=\sum_{\nu \stackrel{i}{\rightarrow} \lambda} v^{-N_{i+\ell}^{r}(\widehat{\nu}, \widehat{\lambda})} \nu, \quad F_{i} \circ \lambda=\sum_{\lambda \stackrel{i}{\rightarrow} \mu} v^{N_{i+\ell}^{l}(\widehat{\lambda}, \widehat{\mu})} \mu .
\end{aligned}
$$

Moreover, the empty $p$-multipartition $\underline{\emptyset}:=(\underbrace{\emptyset, \cdots, \emptyset}_{p})$ is a highest weight vector of weight $\Lambda=\Lambda_{0}+\Lambda_{\ell}+\cdots+\Lambda_{(p-1) \ell}$, and $\widehat{L}(\Lambda):=U_{v}\left(\widehat{\mathfrak{s l}}_{p \ell}\right) \circ \underline{\emptyset}$ is isomorphic to the irreducible highest weight module with highest weight $\Lambda$. 
Proof. For any $x, y \in U_{v}\left(\widehat{\mathfrak{s l}}_{p \ell}\right)$ and $\lambda \in \mathcal{P}$, we have that

$$
\begin{aligned}
x \circ(y \circ \lambda) & =\left(\Theta\left(\Delta^{p-1}(x)\right)\right)\left(\left(\Theta\left(\Delta^{p-1}(y)\right)\right) \lambda\right) \\
& =\left(\Theta\left(\Delta^{p-1}(x)\right) \Theta\left(\Delta^{p-1}(y)\right)\right) \lambda=\left(\Theta\left(\Delta^{p-1}(x) \Delta^{p-1}(y)\right)\right) \lambda \\
& =\left(\Theta\left(\Delta^{p-1}(x y)\right)\right) \lambda=(x y) \circ \lambda,
\end{aligned}
$$

and $1 \circ \lambda=K_{0} \circ \lambda=\lambda$. It follows that the action "o" does define a representation of the algebra $U_{v}\left(\widehat{\mathfrak{s l}}_{p \ell}\right)$ on $\widehat{\mathcal{F}}(\Lambda)$. The remaining part of the lemma can be verified easily.

Therefore, both $\mathcal{F}(\Lambda)$ and $\widehat{\mathcal{F}}(\Lambda)$ are integrable $U_{v}\left(\widehat{\mathfrak{s l}}_{p \ell}\right)$-modules. They are not irreducible. In both cases the empty multipartition $\underline{\emptyset}$ is a highest weight vector of weight $\Lambda=\Lambda_{0}+\Lambda_{\ell}+\cdots+\Lambda_{(p-1) \ell}$, and both $L(\Lambda):=U_{v}\left(\widehat{\mathfrak{s l}}_{p \ell}\right) \underline{\emptyset}$ and $\widehat{L}(\Lambda)$ are isomorphic to the irreducible highest weight module with highest weight $\Lambda$. Let $U_{v}^{\prime}\left(\widehat{\mathfrak{s l}}_{p \ell}\right)$ be the subalgebra of $U_{v}\left(\widehat{\mathfrak{s l}}_{p \ell}\right)$ generated by $E_{i}, F_{i}, K_{h_{i}}^{ \pm 1}, i \in \mathbb{Z} / p \ell \mathbb{Z}$. Let \# be the automorphism of $U_{v}^{\prime}\left(\widehat{\mathfrak{s l}}_{p \ell}\right)$ which is defined on generators by

$$
E_{i}^{\#}:=E_{\ell+i}, F_{i}^{\#}:=F_{\ell+i}, K_{h_{i}}^{\#}:=K_{h_{i+\ell}}, \quad \forall i \in \mathbb{Z} / p \ell \mathbb{Z} .
$$

Now we begin to follow the streamline of the proof of [24, (1.5)]. We first recall some basic facts about crystal bases. Let $\mathcal{A}:=\mathbb{Q}\left[v, v^{-1}\right]$, let $A$ be the ring of rational functions in $\mathbb{Q}(v)$ which do not have a pole at 0 . Write

$$
\mathcal{F}(\Lambda)_{\mathcal{A}}:=\bigoplus_{\lambda \in \mathcal{P}} \mathcal{A} \lambda, \quad \mathcal{F}(\Lambda)_{A}:=\bigoplus_{\lambda \in \mathcal{P}} A \lambda,
$$

and we use similar notations for $\widehat{\mathcal{F}}(\Lambda)$. Let $U_{\mathcal{A}}$ be the Lusztig-Kostant $\mathcal{A}$-form of $U_{v}\left(\widehat{\mathfrak{s l}}_{p \ell}\right)$. Then by [7, (2.7)] we know that both $\mathcal{F}(\Lambda)_{\mathcal{A}}$ and $\widehat{\mathcal{F}}(\Lambda)_{\mathcal{A}}$ are $U_{\mathcal{A}}$-modules.

Let $u_{\Lambda}:=\underline{\emptyset}$, the highest weight vector of weight $\Lambda$ in $\mathcal{F}(\Lambda)$. For each $i \in$ $\{0,1, \cdots, p \ell-1\}$, let $\widetilde{E}_{i}, \widetilde{F}_{i}$ be the Kashiwara operators introduced in [31. Let $L(\Lambda)_{A}$ be the $A$-submodule of $\mathcal{F}(\Lambda)_{A}$ generated by all $\widetilde{F}_{i_{1}} \cdots \widetilde{F}_{i_{k}} u_{\Lambda}$ for all $i_{1}, \cdots, i_{k}$ $\in \mathbb{Z} / p \ell Z$. It is a free $A$-module, and is stable under the action of $\widetilde{E}_{i}$ and $\widetilde{F}_{i}$. The set

$$
\mathbb{B}(\Lambda):=\left\{\widetilde{F}_{i_{1}} \cdots \widetilde{F}_{i_{k}} u_{\Lambda}+v L(\Lambda)_{A} \mid i_{1}, \cdots, i_{k} \in \mathbb{Z} / p \ell \mathbb{Z}\right\} \backslash\{0\}
$$

is a basis of $L(\Lambda)_{A} / v L(\Lambda)_{A}$. In [31], the pair $\left(L(\Lambda)_{A}, \mathbb{B}(\Lambda)\right)$ is called the lower crystal basis at $v=0$ of $L(\Lambda)$.

Following [31, the crystal graph of $L(\Lambda)$ is the edge labelled direct graph whose set of vertices is $\mathbb{B}(\Lambda)$ and whose arrows are given by

$$
b \stackrel{i}{\rightarrow} b^{\prime} \Longleftrightarrow \widetilde{F}_{i} b=b^{\prime} \quad \text { for some } i \in \mathbb{Z} / p \ell \mathbb{Z} .
$$

It is a remarkable fact (39, 77, (2.11)]) that the crystal graph of $L(\Lambda)$ is exactly the same as Kleshchev's good lattice if we use the embedding $L(\Lambda) \subset \mathcal{F}(\Lambda)$. In particular, $\mathbb{B}(\Lambda)$ can be identified with $\mathcal{K}:=\bigsqcup_{n \geq 0} \mathcal{K}_{n}$. Henceforth, we fix such an identification.

Let "-" be the involutive ring automorphism of $U_{v}\left(\widehat{\mathfrak{s l}}_{p \ell}\right)$ which is defined by

$$
\begin{gathered}
\bar{v}:=v^{-1}, \overline{K_{h}}:=K_{-h},\left(h \in P^{\vee}\right), \\
\overline{E_{i}}=E_{i}, \quad \overline{F_{i}}:=F_{i}, i=0,1, \cdots, p \ell-1 .
\end{gathered}
$$


This gives rise to an involution (still denoted by "_") of $L(\Lambda)$. That is, for $x=$ $P \underline{\emptyset} \in L(\Lambda)$, we set $\bar{x}:=\bar{P} \underline{\emptyset}$. By [31, there exists a unique $\mathcal{A}$-basis $\{G(\mu) \mid \mu \in \mathcal{K}\}$ of $L(\Lambda)_{\mathcal{A}}$ such that

(G1) $G(\mu) \equiv \mu\left(\bmod v L(\Lambda)_{A}\right)$,

(G2) $\overline{G(\mu)}=G(\mu)$.

The basis $\{G(\mu)\}_{\mu \in \mathcal{K}}$ is called the lower global crystal basis of $L(\Lambda)$. Let $\lambda \in \mathcal{P}_{n}, \mu \in \mathcal{K}_{n}$. Let $d_{\lambda, \mu}:=\left[\widetilde{S}_{\overrightarrow{\mathrm{Q}}}^{\lambda}: \widetilde{D}_{\overrightarrow{\mathrm{Q}}}^{\mu}\right]$, and let $d_{\lambda, \mu}(v) \in \mathcal{A}$ be such that $G(\mu)=\sum_{\lambda} d_{\lambda, \mu}(v) \lambda$. By a well-known result of Ariki [2], $d_{\lambda, \mu}(1)=d_{\lambda, \mu}$, for any $\lambda \in \mathcal{P}_{n}, \mu \in \mathcal{K}_{n}$.

The following six results can be proved by using exactly the same arguments as in the proof of [24, $(2.2),(3.1),(3.2),(3.3),(3.4),(3.5)]$.

Lemma 4.2. Let $\mathcal{F}(\Lambda)^{\#}$ be the $U_{v}^{\prime}\left(\widehat{\mathfrak{s l}}_{p \ell}\right)$-module which is obtained from $\mathcal{F}(\Lambda)$ by twisting the action by the automorphism \#. Let $\phi$ be the linear map $\mathcal{F}(\Lambda)^{\#} \rightarrow$ $\widehat{\mathcal{F}}(\Lambda)$ which is defined by $\sum_{\lambda} f_{\lambda}(v) \lambda \mapsto \sum_{\lambda} f_{\lambda}(v) \widehat{\lambda}$. Then $\phi$ is a $U_{v}^{\prime}\left(\widehat{\mathfrak{s l}}_{p \ell}\right)$-module isomorphism. Moreover, $\phi(L(\Lambda))=\widehat{L}(\Lambda)$.

Lemma 4.3. Let $U_{v}\left(\widehat{\mathfrak{s l}}_{p \ell}\right)^{-}$be the subalgebra of $U_{v}\left(\widehat{\mathfrak{s l}}_{p \ell}\right)$ generated by $F_{i}, i \in$ $\mathbb{Z} / p \ell \mathbb{Z}$. Then there is a $\mathbb{Q}(v)$-linear automorphism (denoted by \#) on the irreducible $U_{v}\left(\widehat{\mathfrak{s l}}_{p \ell}\right)$-module $L(\Lambda)$ such that

$$
(\underline{\emptyset})^{\#}:=\underline{\emptyset},(P x)^{\#}:=P^{\#} x^{\#}, \quad \forall P \in U_{v}\left(\widehat{\mathfrak{s l}}_{p \ell}\right)^{-}, x \in L(\Lambda) .
$$

Lemma 4.4. For any $x \in L(\Lambda)$, we have $\left(\widetilde{F}_{i} x\right)^{\#}=\widetilde{F}_{\ell+i} x^{\#}$.

Corollary 4.5. Let $\lambda \in \mathcal{K}_{n}$ be a Kleshchev p-multipartition of $n$ with respect to $\left(q, 1, \varepsilon, \varepsilon^{2}, \cdots, \varepsilon^{p-1}\right)$, and let $\underline{\emptyset} \stackrel{r_{1}}{\rightarrow} . \stackrel{r_{2}}{\rightarrow} \ldots \ldots \stackrel{r_{n}}{\rightarrow} \lambda$ be a path from $\underline{\emptyset}$ to $\lambda$ in Kleshchev's good lattice with respect to $\left(q, 1, \varepsilon, \varepsilon^{2}, \cdots, \varepsilon^{p-1}\right)$. Then, the sequence

$$
\underline{\emptyset} \stackrel{\ell+r_{1}}{\rightarrow} \cdot \stackrel{\ell+r_{2}}{\rightarrow} \ldots \ldots \stackrel{\ell+r_{n}}{\rightarrow}
$$

also defines a path in Kleshchev's good lattice with respect to $\left(q, 1, \varepsilon, \varepsilon^{2}, \cdots, \varepsilon^{p-1}\right)$. We denote the endpoint by $\lambda^{\#}$.

Lemma 4.6. For each $\mu \in \mathcal{K}, G(\mu)^{\#}=G\left(\mu^{\#}\right)$.

Lemma 4.7. For any $x \in L(\Lambda)$, we have $\phi\left(\widetilde{F}_{i} x\right)=\widetilde{F}_{\ell+i} \circ \phi(x)$.

Recall that $\widehat{L}(\Lambda)$ is also an irreducible highest weight $U_{v}\left(\widehat{\mathfrak{s l}}_{p \ell}\right)$-module of highest weight $\Lambda$. For any $i_{1}, \cdots, i_{k} \in \mathbb{Z} / p \ell \mathbb{Z}$, it is easy to see $\widetilde{F}_{i_{1}} \cdots \widetilde{F}_{i_{k}} u_{\Lambda} \in v L(\Lambda)_{A}$ if and only if $\widetilde{F}_{i_{1}+\ell} \circ \cdots \circ \widetilde{F}_{i_{k}+\ell} \circ u_{\Lambda} \in v \widehat{L}(\Lambda)_{A}$. By Lemma 4.2 and Lemma 4.7 we know that the lower global crystal basis of $\widehat{L}(\Lambda)$ is parameterized by $\widehat{\mathcal{K}}:=\{\widehat{\mu} \mid \mu \in \mathcal{K}\}$. We denote them by $\{\widehat{G}(\widehat{\mu}) \mid \mu \in \mathcal{K}\}$. For any $\lambda \in \mathcal{P}, \mu \in \mathcal{K}$, let $\widehat{d}_{\lambda, \widehat{\mu}}(v) \in \mathcal{A}$ be such that $\widehat{G}(\widehat{\mu})=\sum_{\lambda} \widehat{d}_{\lambda, \widehat{\mu}}(v) \lambda$. The following three results can be proved by using exactly the same arguments as in the proof of [24, (3.6),(3.7),(3.8)].

Corollary 4.8. For any $\lambda \in \mathcal{P}, \mu \in \mathcal{K}$, we have $\phi(G(\mu))=\widehat{G}(\widehat{\mu})$, and $d_{\lambda, \mu}(v)=$ $\widehat{d}_{\widehat{\lambda}, \widehat{\mu}}(v)$. 
Lemma 4.9. Let $\varphi: L(\Lambda) \rightarrow \widehat{\mathcal{F}}(\Lambda)$ be the map which is defined by $\varphi(x):=\phi\left(x^{\#}\right)$. Then, if specialized at $v=1$, the $\varphi$ is the restriction of the $\widehat{\mathfrak{s l}}_{p \ell}$-module isomorphism $\mathcal{F}(\Lambda)_{\mathbb{Q}} \rightarrow \widehat{\mathcal{F}}(\Lambda)_{\mathbb{Q}}$ given by $\lambda \mapsto \lambda$.

Corollary 4.10. For any $\lambda \in \mathcal{P}, \mu \in \mathcal{K}$, we have $\varphi\left(G\left(\mu^{\#}\right)\right)=\widehat{G}(\widehat{\mu})$ and $d_{\lambda, \mu^{\#}}(1)=$ $\widehat{d}_{\lambda, \widehat{\mu}}(1)$.

Proof of Theorem 3.6. It suffices to show that $\mathrm{h}(\mu)=\mu^{\#}$ for any $\mu \in \mathcal{K}_{n}$. It is well-known that $\left(\widetilde{S}_{\overrightarrow{\mathrm{Q}}}^{\widehat{\lambda}}\right)_{\mathbb{C}(v)} \cong\left(\widetilde{S}_{\overrightarrow{\mathrm{Q}}}^{\lambda}\right)_{\mathbb{C}(v)}^{\sigma}$ (see e.g., [25, (3.7),(5.8)]). Hence in the Grothendieck group of the category of finite-dimensional $\mathcal{H}_{q}(p, n)$-modules, $\left[\widetilde{S}_{\overrightarrow{\mathrm{Q}}}^{\widehat{\lambda}}\right]=$ $\left[\left(\widetilde{S}_{\overrightarrow{\mathrm{Q}}}^{\lambda}\right)^{\sigma}\right]$. By Corollary 4.8, Corollary 4.10 and Ariki's result [2], we deduce that

$$
\begin{aligned}
{\left[\widetilde{S}_{\overrightarrow{\mathrm{Q}}}^{\widehat{\lambda}}: \widetilde{D}_{\overrightarrow{\mathrm{Q}}}^{\mathrm{h}(\mu)}\right] } & =\left[\left(\widetilde{S}_{\overrightarrow{\mathrm{Q}}}^{\lambda}\right)^{\sigma}:\left(\widetilde{D}_{\overrightarrow{\mathrm{Q}}}^{\mu}\right)^{\sigma}\right]=\left[\widetilde{S}_{\overrightarrow{\mathrm{Q}}}^{\lambda}: \widetilde{D}_{\overrightarrow{\mathrm{Q}}}^{\mu}\right]=d_{\lambda, \mu} \\
& =d_{\lambda, \mu}(1)=\widehat{d}_{\widehat{\lambda}, \widehat{\mu}}(1)=d_{\widehat{\lambda}, \mu^{\#}}(1)=d_{\widehat{\lambda}, \mu^{\#}}=\left[\widetilde{S}_{\overrightarrow{\mathrm{Q}}}^{\widehat{\lambda}}: \widetilde{D}_{\overrightarrow{\mathrm{Q}}}^{\mu^{\#}}\right]
\end{aligned}
$$

for any $\lambda \in \mathcal{P}_{n}$. Taking $\hat{\lambda}$ to be $\mathrm{h}(\mu)$ or $\mu^{\#}$, we get that $\mu^{\#} \unlhd \mathrm{h}(\mu)$ and $h(\mu) \unlhd \mu^{\#}$, hence $\mathrm{h}(\mu)=\mu^{\#}$, as required. This completes the proof of Theorem 3.6.

Corollary 4.11. For any $\lambda \in \mathcal{P}_{n}$ and $\mu \in \mathcal{K}_{n}$, $\left[\widetilde{S}_{\overrightarrow{\mathrm{Q}}}^{\lambda}: \widetilde{D}_{\overrightarrow{\mathrm{Q}}}^{\mu}\right]=\left[\widetilde{S}_{\overrightarrow{\mathrm{Q}}}^{\widehat{\lambda}}: \widetilde{D}_{\overrightarrow{\mathrm{Q}}}^{\mathrm{h}(\mu)}\right]$. In particular, $\left[\widetilde{S}_{\overrightarrow{\mathrm{Q}}}^{\widehat{\mu}}: \widetilde{D}_{\overrightarrow{\mathrm{Q}}}^{\mathrm{h}(\mu)}\right]=1$ for any $\mu \in \mathcal{K}_{n}$.

\section{Proof of Theorem 3.8}

In this section, we shall give the proof of Theorem 3.8, Our main tools are Dipper and Mathas's Morita equivalence results ([15]) for Ariki-Koike algebras and their connections with type $A$ affine Hecke algebras. Throughout this section, we keep the same assumptions and notations as in Theorem 3.8. That is, $K=\mathbb{C}, q, \varepsilon \in \mathbb{C}$ be such that $\varepsilon$ is a primitive $p$-th root of unity, $\varepsilon^{k}=q^{\ell}$ is a primitive $d$-th root of unity and $q$ is a primitive $(d \ell)$-th root of unity, and $1<k<p$ is the smallest positive integer such that $\varepsilon^{k} \in\langle q\rangle$.

Let $\mathcal{H}_{q}\left(\mathfrak{S}_{n}\right)$ be the Iwahori-Hecke algebra associated to the symmetric group $\mathfrak{S}_{n}$. Let $K\left[X_{1}^{ \pm 1}, \cdots, X_{n}^{ \pm 1}\right]$ be the ring of Laurent polynomials on $n$ indeterminates $X_{1}, \cdots, X_{n}$.

Definition 5.1. The type $A$ affine Hecke algebra $\mathcal{H}_{n}^{\text {aff }}$ is the $K$-algebra, which as a $K$-linear space is isomorphic to

$$
\mathcal{H}_{q}\left(\mathfrak{S}_{n}\right) \otimes_{K} K\left[X_{1}^{ \pm 1}, \cdots, X_{n}^{ \pm 1}\right] .
$$

The algebra structure is given by requiring that $\mathcal{H}_{q}\left(\mathfrak{S}_{n}\right)$ and $K\left[X_{1}^{ \pm 1}, \cdots, X_{n}^{ \pm 1}\right]$ are subalgebras and that

$$
T_{i} f-{ }^{s_{i}} f T_{i}=(q-1) \frac{f-{ }^{s_{i}} f}{1-X_{i} X_{i+1}^{-1}}, \quad \forall f \in K\left[X_{1}^{ \pm 1}, \cdots, X_{n}^{ \pm 1}\right],
$$

Here $s_{i} \in \mathfrak{S}_{n}$ act on $K\left[X_{1}^{ \pm 1}, \cdots, X_{n}^{ \pm 1}\right]$ by permuting $X_{i}$ and $X_{i+1}$.

Note that the relation (5.2) is equivalent to

$$
\begin{aligned}
& T_{i} X_{i} T_{i}=q X_{i+1}, \quad \forall i \text { with } 1 \leq i<n, \\
& T_{i} X_{j}=X_{j} T_{i}, \quad \forall j \notin\{i, i+1\},
\end{aligned}
$$


Let $Q_{1}, \cdots, Q_{p}$ be elements of $K$. Let $\mathcal{H}(p, n):=\mathcal{H}_{q, Q_{1}, \cdots, Q_{p}}(p, n)$ be the ArikiKoike algebras with parameters $\left\{q, Q_{1}, \cdots, Q_{p}\right\}$. It is well-known that there is a surjective $K$-algebra homomorphism $\varphi: \mathcal{H}_{n}^{\text {aff }} \rightarrow \mathcal{H}(p, n)$ which is defined on generators by

$$
T_{i} \mapsto T_{i}, \quad X_{j} \mapsto L_{j}, \forall 1 \leq i<n, \forall 1 \leq j \leq n,
$$

where $L_{j}:=q^{1-j} T_{j-1} \cdots T_{1} T_{0} T_{1} \cdots T_{j-1}$ (the $j$-th Murphy operator). As a consequence, every simple $\mathcal{H}(p, n)$-module is a simple $\mathcal{H}_{n}^{\text {aff }}$-module.

We shall use Dipper and Mathas's explicit construction of Morita equivalence for Ariki-Koike algebras. To this end, we need some notations and definitions. Let $\left\{s_{1}, s_{2}, \cdots, s_{n-1}\right\}$ be the set of basic transpositions in $\mathfrak{S}_{n}$. A word $w=s_{i_{1}} \cdots s_{i_{k}}$ for $w \in \mathfrak{S}_{n}$ is a reduced expression if $k$ is minimal; in this case we say that $w$ has length $k$ and we write $\ell(w)=k$. Given a reduced expression $s_{i_{1}} \cdots s_{i_{k}}$ for $w \in \mathfrak{S}_{n}$, we write $T_{w}=T_{i_{1}} \cdots T_{i_{k}}$, then $T_{w}$ depends only on $w$ and not on the choice of reduced expression. It is well-known that $\mathcal{H}_{q}\left(\mathfrak{S}_{n}\right)$ is a free module with basis $\left\{T_{w} \mid w \in \mathfrak{S}_{n}\right\}$. For each integer $0 \leq a \leq n$, we define

$$
w_{n-a, a}=\underbrace{\left(s_{n-a} \cdots s_{n-1}\right)}_{a \text { times }} \underbrace{\left(s_{n-a-1} \cdots s_{n-2}\right)}_{a \text { times }} \cdots \underbrace{\left(s_{1} \cdots s_{a}\right)}_{a \text { times }}
$$

if $a \notin\{0, n\}$; or $w_{n-a, a}=1$ if $a \in\{0, n\}$.

Let $s$ be an integer with $1 \leq s \leq p$ and such that

$$
\prod_{1 \leq i \leq s<j \leq p} \prod_{n<a<n}\left(q^{a} Q_{i}-Q_{j}\right) \neq 0
$$

in $K$.

Definition 5.3 ([15]). For each integer $0 \leq b \leq n$, let

$$
\begin{aligned}
u_{n-b}^{-} & :=\prod_{t=1}^{s}\left(L_{1}-Q_{t}\right)\left(L_{2}-Q_{t}\right) \cdots\left(L_{n-b}-Q_{t}\right), \\
u_{b}^{+} & :=\prod_{t=s+1}^{p}\left(L_{1}-Q_{t}\right)\left(L_{2}-Q_{t}\right) \cdots\left(L_{b}-Q_{t}\right), \\
v_{b} & :=u_{n-b}^{-} T_{w_{n-b, b}} u_{b}^{+}, V^{b}:=v_{b} \mathcal{H}(p, n) .
\end{aligned}
$$

Let $\mathcal{H}(s, b)=\mathcal{H}_{q, Q_{1}, \cdots, Q_{s}}(s, b)\left(\right.$ resp., $\left.\mathcal{H}(p-s, n-b)=\mathcal{H}_{q, Q_{s+1}, \cdots, Q_{p}}(p-s, n-b)\right)$ be the Ariki-Koike algebra with parameters $\left\{q, Q_{1}, \cdots, Q_{s}\right\}$ and of size $b$ (resp., with parameters $\left\{q, Q_{s+1}, \cdots, Q_{p}\right\}$ and of size $\left.n-b\right)$. Following [15, let $\hat{\Pi}_{s, b}$ be the functor from $\bmod _{\mathcal{H}(s, b) \otimes \mathcal{H}(p-s, n-b)}$ to $\bmod _{\mathcal{H}(p, n)}$ given by

$$
\hat{\Pi}_{s, b}(X):=X \otimes_{\mathcal{H}(s, b) \otimes \mathcal{H}(p-s, n-b)} V^{b}, \forall X \in \bmod _{\mathcal{H}(s, b) \otimes \mathcal{H}(p-s, n-b)} .
$$

Note that here the left $(\mathcal{H}(s, b) \otimes \mathcal{H}(p-s, n-b))$-action on $V^{b}$ is well-defined because of the following very useful result from [15, (3.4)].

Lemma 5.4 ([15, (3.4)]). Suppose that $0 \leq b \leq n$.

$$
T_{i} v_{b}= \begin{cases}v_{b} T_{i+b}, & \text { if } 1 \leq i<n-b, \\ v_{b} T_{i-n+b}, & \text { if } n-b<i \leq n .\end{cases}
$$


(ii)

$$
L_{k} v_{b}= \begin{cases}v_{b} L_{k+b}, & \text { if } 1 \leq k \leq n-b, \\ v_{b} L_{k-n+b}, & \text { if } n-b+1 \leq k \leq n .\end{cases}
$$

Let $\overrightarrow{\mathrm{Q}}_{1}:=\left(Q_{1}, \cdots, Q_{s}\right), \overrightarrow{\mathrm{Q}}_{2}:=\left(Q_{s+1}, \cdots, Q_{p}\right)$. Let $\overrightarrow{\mathrm{Q}}:=\left(\overrightarrow{\mathrm{Q}}_{1}, \overrightarrow{\mathrm{Q}}_{2}\right)=$ $\left(Q_{1}, \cdots, Q_{p}\right)$. Suppose that $D_{\overrightarrow{\mathrm{Q}}_{1}}^{\lambda^{[1]}} \neq 0$ (resp., $D_{\overrightarrow{\mathrm{Q}}_{2}}^{\lambda^{[2]}} \neq 0$ ) is an irreducible $\mathcal{H}(s, b)$ module (resp., $\mathcal{H}(p-s, n-b)$-module), where $\lambda^{[1]}$ (resp., $\lambda^{[2]}$ ) is an $s$-multipartition of $b$ (resp., $(p-s)$-multipartition of $n-b)$. Let $\lambda:=\left(\lambda^{[1]}, \lambda^{[2]}\right)$ (concatenation of ordered tuples), which is a $p$-multipartition of $n$. By [15], $D_{\overrightarrow{\mathrm{Q}}}^{\lambda} \neq 0$ is an irreducible $\mathcal{H}(p, n)$-module, and

$$
\hat{\Pi}_{s, b}\left(D_{\overrightarrow{\mathrm{Q}}_{1}}^{\lambda^{[1]}} \otimes D_{\overrightarrow{\mathrm{Q}}_{2}}^{\lambda^{[2]}}\right)=\left(D_{\overrightarrow{\mathrm{Q}}_{1}}^{\lambda^{[1]}} \otimes D_{\overrightarrow{\mathrm{Q}}_{2}}^{\lambda^{[2]}}\right) \otimes_{\mathcal{H}(s, b) \otimes \mathcal{H}(p-s, n-b)} V^{b} \cong D_{\overrightarrow{\mathrm{Q}}}^{\lambda} .
$$

Let $\mathcal{H}_{b}^{\text {aff }}$ (resp., $\mathcal{H}_{n-b}^{\text {aff }}$ ) be the standard parabolic subalgebra of $\mathcal{H}_{n}^{\text {aff }}$ generated by $T_{1}, \cdots, T_{b-1}, X_{1}, \cdots, X_{b}$ (resp., by $T_{b+1}, \cdots, T_{n-1}, X_{b+1}, \cdots, X_{n}$ ). Then $D_{\overrightarrow{\mathrm{Q}}_{1}}^{\lambda^{[1]}}$ (resp., $D_{\overrightarrow{\mathrm{Q}}_{2}}^{\lambda^{[2]}}$ ) naturally becomes an irreducible $\mathcal{H}_{b}^{\text {aff }}$-module (resp., $\mathcal{H}_{n-b}^{\text {aff }}$-module). We have that

Proposition 5.5. There is an $\mathcal{H}_{n}^{\text {aff }}$-module isomorphism

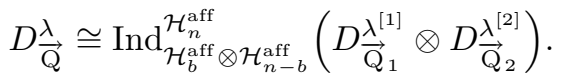

Proof. By our previous discussion, it suffices to show that

$$
\begin{aligned}
&\left(D_{\overrightarrow{\mathrm{Q}}_{1}}^{\lambda^{[1]}} \otimes D_{\overrightarrow{\mathrm{Q}}_{2}}^{\lambda^{[2]}}\right) \otimes_{\mathcal{H}_{b}^{\text {aff }}} \otimes \mathcal{H}_{n-b}^{\text {aff }} \mathcal{H}_{n}^{\text {aff }} \\
& \cong\left(D_{\overrightarrow{\mathrm{Q}}_{1}}^{\lambda^{[1]}} \otimes D_{\overrightarrow{\mathrm{Q}}_{2}}^{\lambda^{[2]}}\right) \otimes_{\mathcal{H}(s, b) \otimes \mathcal{H}(p-s, n-b)} v_{b} \mathcal{H}(p, n),
\end{aligned}
$$

where $v_{b} \mathcal{H}(p, n)$ is regarded as a right $\mathcal{H}_{n}^{\text {aff }}$-module via the natural surjective homomorphism $\varphi: \mathcal{H}_{n}^{\text {aff }} \rightarrow \mathcal{H}(p, n)$.

In fact, by Lemma 5.4, it is easy to see that the following map

$$
(x \otimes y) \otimes_{\mathcal{H}_{b}^{\text {aff }} \otimes \mathcal{H}_{n-b}^{\text {aff }}} h \mapsto(x \otimes y) \otimes_{\mathcal{H}(s, b) \otimes \mathcal{H}(p-s, n-b)} v_{b} h
$$

extends naturally to a well-defined surjective right $\mathcal{H}_{n}^{\text {aff }}$-module homomorphism. Now comparing their dimensions (see [15, (4.8)]), we proved the theorem.

Now we suppose that $\mathrm{Q}=\mathrm{Q}_{1} \sqcup \mathrm{Q}_{2} \sqcup \cdots \sqcup \mathrm{Q}_{\kappa}$ (disjoint union) such that $Q_{i}, Q_{j}$ are in the same $q$-orbit only if $Q_{i}, Q_{j} \in \mathrm{Q}_{c}$ for some $1 \leq c \leq \kappa$. For each integer $i$ with $1 \leq i \leq \kappa$, let $D_{\overrightarrow{\mathrm{Q}}_{i}}^{\lambda^{[i]}} \neq 0$ be an irreducible $\mathcal{H}\left(p_{i}, b_{i}\right)$-module, where $p_{i}=\left|\overrightarrow{\mathrm{Q}}_{i}\right|$, $\lambda^{[i]}$ is a $p_{i}$-multipartition of $b_{i}, \sum_{i=1}^{\kappa} b_{i}=n$. Let $\lambda:=\left(\lambda^{[1]}, \cdots, \lambda^{[\kappa]}\right)$ (concatenation of ordered tuples), which is a $p$-multipartition of $n$.

Corollary 5.6. With the above assumptions and notations, we have an $\mathcal{H}_{n}^{\text {aff }}$-module isomorphism

$$
D_{\overrightarrow{\mathrm{Q}}}^{\lambda} \cong \operatorname{Ind}_{\mathcal{H}_{b_{1}}^{\text {aff }}}^{\mathcal{H}_{\text {aff }}^{\text {aff }} \cdots \otimes \mathcal{H}_{b_{\kappa}}^{\text {aff }}}\left(D_{\overrightarrow{\mathrm{Q}}_{1}}^{\lambda^{[1]}} \otimes \cdots \otimes D_{\overrightarrow{\mathrm{Q}}_{\kappa}}^{\lambda^{[\kappa]}}\right)
$$

Proof. This follows from Proposition [5.5] and the associativity of tensor product induction functor. 
Now we return to our setup in Theorem 3.8 . For each $1 \leq i \leq k$, we set $\overrightarrow{\mathrm{Q}}_{i}=$ $\left(\varepsilon^{i-1}, \varepsilon^{k+i-1}, \cdots, \varepsilon^{(d-1) k+i-1}\right) . \mathrm{Q}=\mathrm{Q}_{1} \sqcup \cdots \sqcup \mathrm{Q}_{k}$ is a partition of the parameters set $\mathrm{Q}$ into different $q$-orbits. Let $\overrightarrow{\mathrm{Q}}=\left(\overrightarrow{\mathrm{Q}}_{1}, \overrightarrow{\mathrm{Q}}_{2}, \cdots, \overrightarrow{\mathrm{Q}}_{k}\right)$ (concatenation of ordered tuples). For each $p$-multipartition $\lambda=\left(\lambda^{(1)}, \cdots, \lambda^{(p)}\right)$ of $n$, we write

$$
\lambda^{[i]}=\left(\lambda^{((i-1) d+1)}, \lambda^{((i-1) d+2)}, \cdots, \lambda^{(i d)}\right), \text { for } i=1,2, \cdots, k,
$$

and recall $\theta$ is the map $\lambda \mapsto\left(\lambda^{[1]}, \cdots, \lambda^{[k]}\right)$. Let $n_{i}=\left|\lambda^{[i]}\right|$ for each $1 \leq i \leq k$.

Proof of Theorem 3.8. We define

$$
\overrightarrow{\mathrm{Q}}_{1}^{*}:=\left(\varepsilon^{k}, \varepsilon^{2 k}, \cdots, \varepsilon^{(d-1) k}, 1\right) .
$$

By Lemma 2.4, we have that

$$
\left(\widetilde{D}_{\overrightarrow{\mathrm{Q}}}^{\lambda}\right)^{\sigma} \cong \widetilde{D}_{\left(\overrightarrow{\mathrm{Q}}_{2}, \overrightarrow{\mathrm{Q}}_{3}, \cdots, \overrightarrow{\mathrm{Q}}_{k}, \overrightarrow{\mathrm{Q}}_{1}^{*}\right)}
$$

Applying Corollary [5.6, we get that

$$
\begin{aligned}
& \left(\widetilde{D}_{\overrightarrow{\mathrm{Q}}}^{\lambda}\right)^{\sigma} \cong \widetilde{D}_{\left(\overrightarrow{\mathrm{Q}}_{2}, \overrightarrow{\mathrm{Q}}_{3}, \cdots, \overrightarrow{\mathrm{Q}}_{k}, \overrightarrow{\mathrm{Q}}_{1}^{*}\right)}^{\lambda}
\end{aligned}
$$

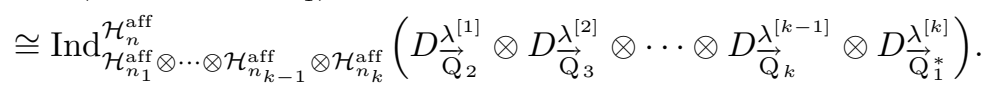

By [44, (5.12)], the right-hand side module has the same composition factors as

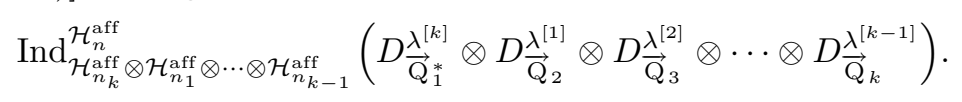

In particular, as both modules are irreducible, these two modules are in fact isomorphic to each other. Therefore,

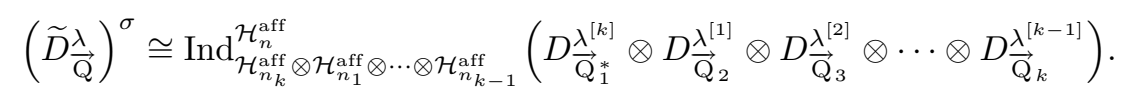

Now again by Lemma 2.4 ,

$$
D_{\overrightarrow{\mathrm{Q}}_{1}^{*}}^{\lambda^{[k]}} \cong\left(\widetilde{D}_{\overrightarrow{\mathrm{Q}}_{1}}^{\lambda^{[k]}}\right)^{\sigma^{\prime}}
$$

where $\sigma^{\prime}$ denotes the $K$-algebra automorphism of the Ariki-Koike algebra

$$
\mathcal{H}_{q}\left(d, n_{k}\right):=\mathcal{H}_{q, 1, \varepsilon^{\prime}, \cdots,\left(\varepsilon^{\prime}\right)^{d-1}}\left(d, n_{k}\right)
$$

(where $\varepsilon^{\prime}=\varepsilon^{k}$ ) which is defined on generators by $\sigma\left(T_{0}\right)=\varepsilon^{\prime} T_{0}, \sigma\left(T_{i}\right)=T_{i}$, for $i=1,2, \cdots, n_{k}-1$. By Theorem 3.6 and Corollary 3.7, we deduce that

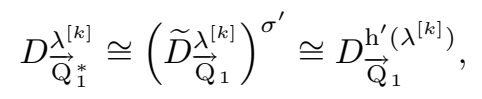

where $\mathrm{h}^{\prime}$ is as defined in Corollary 3.7, Therefore,

$$
\begin{aligned}
& \left(\widetilde{D}_{\overrightarrow{\mathrm{Q}}}^{\lambda}\right)^{\sigma} \cong \operatorname{Ind}_{\mathcal{H}_{n_{k}}^{\text {aff }}}^{\mathcal{H}_{n_{n_{1}}}^{\text {aff }}} \mathcal{H}_{n_{1}}^{\text {aff }} \otimes \otimes \mathcal{H}_{n_{k-1}}^{\text {aff }}\left(D_{\overrightarrow{\mathrm{Q}}_{1}^{*}}^{\lambda^{[k]}} \otimes D_{\overrightarrow{\mathrm{Q}}_{2}}^{\lambda^{[1]}} \otimes D_{\overrightarrow{\mathrm{Q}}_{3}}^{\lambda^{[2]}} \otimes \cdots \otimes D_{\overrightarrow{\mathrm{Q}}_{k}}^{\lambda^{[k-1]}}\right) \\
& \cong \operatorname{Ind}_{\mathcal{H}_{n_{k}}^{\text {aff }}}^{\mathcal{H}_{k}^{\text {aff }}} \otimes \mathcal{H}_{n_{1}}^{\text {aff }} \otimes \cdots \otimes \mathcal{H}_{n_{k-1}}^{\text {aff }}\left(D_{\overrightarrow{\mathrm{Q}}_{1}}^{\mathrm{h}^{\prime}\left(\lambda^{[k]}\right)} \otimes D_{\overrightarrow{\mathrm{Q}}_{2}}^{\lambda^{[1]}} \otimes D_{\overrightarrow{\mathrm{Q}}_{3}}^{\lambda^{[2]}} \otimes \cdots \otimes D_{\overrightarrow{\mathrm{Q}}_{k}}^{\lambda^{[k-1]}}\right) \\
& \cong \widetilde{D}_{\overrightarrow{\mathrm{Q}}}^{\left(\mathrm{h}^{\prime}\left(\lambda^{[k]}\right), \lambda^{[1]}, \cdots, \lambda^{[k-1]}\right)} \text {. }
\end{aligned}
$$

It follows that

$$
\theta(\mathrm{h}(\lambda))=\left(\mathrm{h}^{\prime}\left(\lambda^{[k]}\right), \lambda^{[1]}, \cdots, \lambda^{[k-1]}\right),
$$

as required. This completes the proof of Theorem 3.8 . 


\section{Closed formula for the number of Simple $\mathcal{H}_{q}(p, p, n)$-Modules}

The purpose of this section is to give the second two main results (Theorem 6.10 and Theorem 6.17) of this paper, which yield an explicit formula for the number of simple modules over the cyclotomic Hecke algebra of type $G(p, p, n)$ in the nonseparated case. Note that in the separated case one can easily write an explicit formula by using the result [25, (5.7)]. In the non-separated case we shall apply Theorem [3.6 and Theorem 3.8 as well as Naito and Sagaki's work (40, 41]) on Lakshmibai-Seshadri paths fixed by diagram automorphisms.

Recall our definitions of $K_{n}$ and $\mathrm{h}$ in the second paragraph of Section 3.

Definition 6.1. For each $\lambda \in K_{n}$, let

$$
o_{\mathrm{h}}(\lambda):=\min \left\{1 \leq m \leq p \mid \mathrm{h}^{m}(\lambda)=\lambda\right\} .
$$

For each integer $m$ with $1 \leq m \leq p$, we define

$$
\begin{aligned}
& \widetilde{\Sigma}(m):=\left\{\lambda \in K_{n} \mid \mathrm{h}^{m}(\lambda)=\lambda\right\}, \widetilde{N}(m):=\# \widetilde{\Sigma}(m), \\
& N(m):=\#\left\{\lambda \in K_{n} \mid o_{\mathrm{h}}(\lambda)=m\right\} .
\end{aligned}
$$

We use the notation $\# \operatorname{Irr}\left(\mathcal{H}_{q}(p, p, n)\right)$ (resp., \# $\operatorname{Irr}\left(\mathcal{H}_{q}(p, n)\right)$ ) to denote the number of simple $\mathcal{H}_{q}(p, p, n)$-modules (resp., simple $\mathcal{H}_{q}(p, n)$-modules). By Lemma 3.1. we know that

$$
\begin{aligned}
\# \operatorname{Irr}\left(\mathcal{H}_{q}(p, p, n)\right)=\frac{1}{p}\left\{\# \operatorname{Irr}\left(\mathcal{H}_{q}(p, n)\right)-\sum_{1 \leq m<p, m \mid p} N(m)\right\} \\
+\sum_{1 \leq m<p, m \mid p} \frac{N(m)}{m} \frac{p}{m} .
\end{aligned}
$$

Note that by [7, the number \# $\operatorname{Irr}\left(\mathcal{H}_{q}(p, n)\right)$ is explicitly known. Therefore, to get a formula for $\# \operatorname{Irr}\left(\mathcal{H}_{q}(p, p, n)\right)$, it suffices to derive a formula for $N(\widetilde{m})$ for each integer $1 \leq \widetilde{m}<p$ satisfying $\widetilde{m} \mid p$.

Let $\mu$ be the Möbius function $\mu: \mathbb{N} \rightarrow\{0,1,-1\}$ which is given by

$$
\mu(a)= \begin{cases}1, & \text { if } a=1, \\ (-1)^{s}, & \text { if } a=p_{1} \cdots p_{s}, \text { where }\left\{p_{i}\right\}_{1 \leq i \leq s} \text { are } \\ 0 & \text { otherwise different prime numbers }\end{cases}
$$

Since $\tilde{N}(m)=\sum_{1 \leq a \leq m, a \mid m} N(a)$, it follows from the Möbius inversion formula that

$$
N(\widetilde{m})=\sum_{1 \leq m \leq \widetilde{m}, m \mid \widetilde{m}} \mu(\widetilde{m} / m) \tilde{N}(m)
$$

Therefore, it suffices to derive a formula for $\tilde{N}(m)$ for each integer $1 \leq m<p$ satisfying $m \mid p$. To this end, we have to use Naito and Sagaki's work ([40, [41]) on Lakshmibai-Seshadri paths fixed by diagram automorphisms.

For the moment, we assume the following setup. That is, $K=\mathbb{C}, q, \varepsilon \in \mathbb{C}$ be such that $\varepsilon=q^{\ell}$ is a primitive $p$-th root of unity, and $q$ is a primitive $p \ell$-th root of unity. We identify $K_{n}$ with $\mathcal{K}_{n}$, the set of Kleshchev $p$-multipartitions with respect to $\left\{q, 1, \varepsilon, \cdots, \varepsilon^{p-1}\right\}$. Let $\mathfrak{g}$ be the Kac-Moody algebra over $\mathbb{C}$ associated to a symmetrizable generalized Cartan matrix $\left(a_{i, j}\right)_{i, j \in I}$ of finite size. Let $\mathfrak{h}$ be its Cartan subalgebra, and $W \subset \mathrm{GL}\left(\mathfrak{h}^{*}\right)$ its Weyl group. Let $\left\{\alpha_{i}^{\vee}\right\}_{0 \leq i \leq n-1}$ be the 
set of simple coroots in $\mathfrak{h}$. Let $\mathcal{X}:=\left\{\Lambda \in \mathfrak{h}^{*} \mid \Lambda\left(\alpha_{i}^{\vee}\right) \in \mathbb{Z}, \forall 0 \leq i<n\right\}$ be the weight lattice. Let $\mathcal{X}^{+}:=\left\{\Lambda \in \mathcal{X} \mid \Lambda\left(\alpha_{i}^{\vee}\right) \geq 0, \forall 0 \leq i<n\right\}$ be the set of integral dominant weights. Let $\mathcal{X}_{\mathbb{R}}:=\mathcal{X} \otimes_{\mathbb{Z}} \mathbb{R}$, where $\mathbb{R}$ is the real number field. Assume that $\Lambda \in \mathcal{X}^{+}$. P. Littelmann introduced (33, 34]) the notion of Lakshmibai-Seshadri paths (L-S paths for short) of class $\Lambda$, which are piecewise linear, continuous maps $\pi:[0,1] \rightarrow \mathcal{X}_{\mathbb{R}}$ parameterized by pairs $(\underline{\nu}, \underline{a})$ of a sequence $\underline{\nu}: \nu_{1}>\nu_{2}>\cdots>\nu_{s}$ of elements of $W \Lambda$, where $>$ is the "relative Bruhat order" (see [34, Section 4]) on $W \Lambda$, and a sequence $\underline{a}: 0=a_{0}<a_{1}<\cdots<a_{s}=1$ of rational numbers with a certain condition, called the chain condition. The set $\mathbb{B}(\Lambda)$ of all L-S paths of class $\Lambda$ is called the path model for the irreducible integrable highest weight module $L(\Lambda)$ of highest weight $\Lambda$ over $\mathfrak{g}$. It is a remarkable fact that $\mathbb{B}(\Lambda)$ has a canonical crystal structure which is isomorphic to the crystal associated to the irreducible integrable highest weight module of highest weight $\Lambda$ over the quantum affine algebra $U_{v}^{\prime}(\mathfrak{g})$.

Now let $\mathfrak{g}=\widehat{\mathfrak{s l}}_{p \ell}$, the affine Kac-Moody algebra of type $A_{p \ell-1}^{(1)}$. The generalized Cartan matrix $\left(a_{i, j}\right)_{i, j \in I}$ of $\mathfrak{g}$ was indexed by the finite set $I:=\mathbb{Z} / p \ell \mathbb{Z}$. Let $1 \leq m<$ $p$ be an integer satisfying $m \mid p$. Let $\omega: I \rightarrow I$ be an automorphism of order $p / m$ defined by $\bar{i}=i+p \ell \mathbb{Z} \mapsto \bar{i}-\overline{m \ell}=i-m \ell+p \ell \mathbb{Z}$ for any $\bar{i} \in I$. Clearly $\omega$ is a Dynkin diagram automorphism in the sense of [40, $\S 1.2]$ (i.e., satisfying $a_{\omega(i), \omega(j)}=a_{i, j}$, $\forall i, j \in I$ ). By [18, $\omega$ induces a Lie algebra automorphism (which is called the diagram outer automorphism) $\omega \in \operatorname{Aut}(\mathfrak{g})$ of order $p / m$ and a linear automorphism $\omega^{*} \in \mathrm{GL}\left(\mathfrak{h}^{*}\right)$ of order $p / m$.

Following [17] and [40, §1.3], we set $c_{i, j}:=\sum_{t=0}^{N_{j}-1} a_{i, \omega^{t}(j)}$, where $N_{j}:=\#\left\{\omega^{t}(i) \mid\right.$ $t \geq 0\}, i, j \in I$. We choose a complete set $\widehat{I}$ of representatives of the $\omega$-orbits in $I$, and set $\check{I}:=\left\{i \in \widehat{I} \mid c_{i, i}>0\right\}$. We put $\hat{a}_{i, j}:=2 c_{i, j} / c_{j}$ for $i, j \in \widehat{I}$, where $c_{i}:=c_{i i}$ if $i \in \check{I}$, and $c_{i}:=2$ otherwise. Then $\left(\hat{a}_{i, j}\right)_{i, j \in \widehat{I}}$ is a symmetrizable Borcherds-Cartan matrix in the sense of $\left[\right.$ ], and (if $\check{I} \neq \emptyset$ ) its submatrix $\left(\hat{a}_{i, j}\right)_{i, j \in \check{I}}$ is a generalized Cartan matrix of affine type. Let $\widehat{\mathfrak{g}}$ be the generalized Kac-Moody algebra over $\mathbb{C}$ associated to $\left(\hat{a}_{i, j}\right)_{i, j \in \widehat{I}}$, with Cartan subalgebra $\widehat{\mathfrak{h}}$, Chevalley generators $\left\{\hat{x}_{i}, \hat{y}_{i}\right\}_{i \in \hat{I}}$. The orbit Lie algebra $\check{\mathfrak{g}}$ is defined to be the subalgebra of $\widehat{\mathfrak{g}}$ generated by $\widehat{\mathfrak{h}}$ and $\hat{x}_{i}, \hat{y}_{i}$ for $i \in \check{I}$, which is a usual Kac-Moody algebra.

Lemma 6.4. With the above assumptions and notations, we have that

$$
\check{\mathfrak{g}}= \begin{cases}\widehat{\mathfrak{s l}}_{m \ell}, & \text { if } m \ell>1, \\ \mathbb{C}, & \text { if } m=\ell=1 .\end{cases}
$$

Proof. This follows from direct verification.

We define $\left(\mathfrak{h}^{*}\right)^{\circ}:=\left\{\Lambda \in \mathfrak{h}^{*} \mid \omega^{*}(\Lambda)=\Lambda\right\} . \widetilde{W}:=\left\{w \in W \mid \omega^{*} w=w \omega^{*}\right\}$. To distinguish from the objects for $\mathfrak{g}$, the objects for the orbit Lie algebra $\mathfrak{\mathfrak { g }}$ will always have the symbol "V" on the head. For example, $\check{\mathfrak{h}}$ denotes the Cartan subalgebra of $\check{\mathfrak{g}}, \check{W}$ the Weyl group of $\check{\mathfrak{g}},\left\{\check{\Lambda}_{i}\right\}_{0 \leq i \leq m \ell-1}$ the set of fundamental dominant weights in $\check{\mathfrak{h}}^{*}$. There exist a linear automorphism $P_{\omega}^{*}: \check{\mathfrak{h}}^{*} \rightarrow\left(\mathfrak{h}^{*}\right)^{\circ}$ and a group isomorphism $\Theta: \check{W} \rightarrow \widetilde{W}$ such that $\Theta(\check{w})=P_{\omega}^{*} \check{w}\left(P_{\omega}^{*}\right)^{-1}$ for each $w \in \check{W}$. By [18, $\S 6.5$ ], for each $0 \leq i<m \ell$,

$$
P_{\omega}^{*}\left(\check{\Lambda}_{i}\right)=\Lambda_{i}+\Lambda_{i+m \ell}+\Lambda_{i+2 m \ell}+\cdots+\Lambda_{i+(p-m) \ell}+C \delta
$$


where $C \in \mathbb{Q}$ is some constant depending on $\omega, \delta$ denotes the null root of $\mathfrak{g}$. Let $\check{\Lambda}=\check{\Lambda}_{0}+\check{\Lambda}_{\ell}+\check{\Lambda}_{2 \ell}+\cdots+\check{\Lambda}_{(m-1) \ell}$. Let $\Lambda:=\Lambda_{0}+\Lambda_{\ell}+\Lambda_{2 \ell}+\cdots+\Lambda_{(p-1) \ell}$. Then it follows that $P_{\omega}^{*}(\check{\Lambda})=\Lambda+C^{\prime} \delta$, for some $C^{\prime} \in \mathbb{Q}$.

Let $\mathbb{B}(\Lambda)$ (resp., $\mathbb{B}\left(P_{\omega}^{*}(\check{\Lambda})\right)$ ) be the set of all L-S paths of class $\Lambda$ (resp., of class $P_{\omega}^{*}(\check{\Lambda})$ ). Let $\pi_{\Lambda}$ (resp., $\pi_{P_{\omega}^{*}(\check{\Lambda})}$ ) be the straight path joining 0 and $\Lambda$ (resp., 0 and $\left.P_{\omega}^{*}(\check{\Lambda})\right)$. Let $\widetilde{E}_{i}, \widetilde{F}_{i}$ denote the raising root operator and the lowering root operator (see [33] and [34]) with respect to the simple root $\alpha_{i}$.

Lemma 6.5. The map which sends $\pi_{P_{\omega}^{*}(\check{\Lambda})}$ to $\pi_{\Lambda}$ extends to a bijection $\beta$ from $\mathbb{B}\left(P_{\omega}^{*}(\check{\Lambda})\right)$ onto $\mathbb{B}(\Lambda)$ such that

$$
\beta\left(\widetilde{F}_{i_{1}} \cdots \widetilde{F}_{i_{s}} \pi_{P_{\omega}^{*}(\check{\Lambda})}\right)=\widetilde{F}_{i_{1}} \cdots \widetilde{F}_{i_{s}} \pi_{\Lambda}
$$

for any $i_{1}, \cdots, i_{s} \in \mathbb{Z} / p \ell \mathbb{Z}$.

Proof. This follows from the fact that $P_{\omega}^{*}(\check{\Lambda})-\Lambda \in \mathbb{Q} \delta$ and the definitions of $\mathbb{B}\left(P_{\omega}^{*}(\check{\Lambda})\right)$ and $\mathbb{B}(\Lambda)$.

Henceforth we shall identify $\mathbb{B}\left(P_{\omega}^{*}(\check{\Lambda})\right)$ with $\mathbb{B}(\Lambda)$. The action of $\omega^{*}$ on $\mathfrak{h}^{*}$ naturally extends to the set $\mathbb{B}\left(P_{\omega}^{*}(\check{\Lambda})\right)$ (and hence to the set $\mathbb{B}(\Lambda)$ ). By [41, (3.1.1)], if $\widetilde{F}_{i_{1}} \widetilde{F}_{i_{2}} \cdots \widetilde{F}_{i_{s}} \pi_{\Lambda} \in \mathbb{B}(\Lambda)$, then

$$
\omega^{*}\left(\widetilde{F}_{i_{1}} \widetilde{F}_{i_{2}} \cdots \widetilde{F}_{i_{s}} \pi_{\Lambda}\right)=\widetilde{F}_{i_{1}+m \ell} \widetilde{F}_{i_{2}+m \ell} \cdots \widetilde{F}_{i_{s}+m \ell} \pi_{\Lambda} .
$$

We denote by $\mathbb{B}^{\circ}(\Lambda)$ the set of all L-S paths of class $\Lambda$ that are fixed by $\omega^{*}$. For $\check{\mathfrak{g}}$, we denote by $\widetilde{e}_{i}, \widetilde{f}_{i}$ the raising root operator and the lowering root operator with respect to the simple root $\alpha_{i}$. Let $\pi_{\check{\Lambda}}$ be the straight path joining 0 and $\check{\Lambda}$. By [40, (4.2)], the linear map $P_{\omega}^{*}$ naturally extends to a map from $\check{\mathbb{B}}(\check{\Lambda})$ to $\mathbb{B}^{\circ}(\Lambda)$ such that if $\widetilde{f}_{i_{1}} \widetilde{f}_{i_{2}} \cdots \widetilde{f}_{i_{s}} \pi_{\check{\Lambda}} \in \check{\mathbb{B}}(\check{\Lambda})$, then

$$
\begin{aligned}
P_{\omega}^{*}\left(\widetilde{f}_{i_{1}} \widetilde{f}_{i_{2}} \cdots \widetilde{f}_{i_{s}} \pi_{\check{\Lambda}}\right)= & \widetilde{F}_{i_{1}} \widetilde{F}_{i_{1}+m \ell} \cdots \widetilde{F}_{i_{1}+(p-m) \ell} \widetilde{F}_{i_{2}} \widetilde{F}_{i_{2}+m \ell} \cdots \widetilde{F}_{i_{2}+(p-m) \ell} \cdots \\
& \widetilde{F}_{i_{s}} \widetilde{F}_{i_{s}+m \ell} \cdots \widetilde{F}_{i_{s}+(p-m) \ell} \pi_{\Lambda} .
\end{aligned}
$$

Lemma 6.7 ([40, $(4.2),(4.3)]) \cdot \mathbb{B}^{\circ}(\Lambda)=P_{\omega}^{*}(\check{\mathbb{B}}(\check{\Lambda}))$.

Note that both $\check{\mathbb{B}}(\check{\Lambda})$ and $\mathbb{B}(\Lambda)$ have a canonical crystal structure with the raising and lowering root operators playing the role of Kashiwara operators. They are isomorphic to the crystals associated to the irreducible integrable highest weight modules $\check{L}(\check{\Lambda})$ of highest weight $\check{\Lambda}$ over $U_{v}^{\prime}(\check{\mathfrak{g}})$ and $L(\Lambda)$ of highest weight $\Lambda$ over $U_{v}^{\prime}(\mathfrak{g})$ respectively. Henceforth, we identify them without further comments. Let $v_{\check{\Lambda}}\left(\right.$ resp., $\left.v_{\Lambda}\right)$ denote the unique highest weight vector of highest weight $\check{\Lambda}$ (resp., of highest weight $\Lambda$ ) in $\check{\mathbb{B}}(\check{\Lambda})$ (resp., in $\mathbb{B}(\Lambda)$ ). Therefore, by (6.6) and Lemma 6.7, we get that

Corollary 6.8. With the above assumptions and notations, there is an injection $\eta$ from the set $\breve{\mathbb{B}}(\check{\Lambda})$ of crystal bases to the set $\mathbb{B}(\Lambda)$ of crystal bases such that

$$
\begin{array}{r}
\eta\left(\widetilde{f}_{i_{1}} \widetilde{f}_{i_{2}} \cdots \widetilde{f}_{i_{s}} v_{\check{\Lambda}}\right) \equiv \widetilde{F}_{i_{1}} \widetilde{F}_{i_{1}+m \ell} \cdots \widetilde{F}_{i_{1}+(p-m) \ell} \widetilde{F}_{i_{2}} \widetilde{F}_{i_{2}+m \ell} \cdots \widetilde{F}_{i_{2}+(p-m) \ell} \cdots \\
\widetilde{F}_{i_{s}} \widetilde{F}_{i_{s}+m \ell} \cdots \widetilde{F}_{i_{s}+(p-m) \ell} v_{\Lambda} \quad\left(\bmod v L(\Lambda)_{A}\right),
\end{array}
$$

and the image of $\eta$ consists of all crystal basis element $\widetilde{F}_{i_{1}} \cdots \widetilde{F}_{i_{t}} v_{\Lambda}+v L(\Lambda)_{A}$ satisfying $\widetilde{F}_{i_{1}} \cdots \widetilde{F}_{i_{t}} v_{\Lambda} \equiv \widetilde{F}_{i_{1}+m \ell} \cdots \widetilde{F}_{i_{t}+m \ell} v_{\Lambda}\left(\bmod v L(\Lambda)_{A}\right)$. 
We translate the language of crystal bases into the language of Kleshchev multipartitions, we get the following combinatorial result, which seems to be of independent interest.

Corollary 6.9. Let $\mathrm{h}$ be as in Theorem 3.6. Let $\varepsilon^{\prime}:=\varepsilon^{p / m}$. Let $q^{\prime}=\sqrt[\ell]{\varepsilon^{\prime}}$, which is a primitive $m \ell$-th root of unity. Then there exists a bijection $\eta: \check{\lambda} \mapsto \lambda$ from the set of Kleshchev m-multipartitions $\check{\lambda}$ of $n m / p$ with respect to $\left(q^{\prime}, 1, \varepsilon^{\prime}, \varepsilon^{\prime 2}, \cdots,\left(\varepsilon^{\prime}\right)^{m-1}\right)$ onto the set of Kleshchev p-multipartitions $\lambda$ of $n$ with respect to $\left(q, 1, \varepsilon, \varepsilon^{2}\right.$, $\cdots, \varepsilon^{p-1}$ ) satisfying $\mathrm{h}^{m}(\lambda)=\lambda$, such that if

$$
\underbrace{(\emptyset, \cdots, \emptyset)}_{m} \stackrel{r_{1}}{\rightarrow} \cdot \stackrel{r_{2}}{\rightarrow} \cdots \cdots \stackrel{r_{s}}{\rightarrow} \check{\lambda}
$$

is a path from $\underbrace{(\emptyset, \cdots, \emptyset)}_{m}$ to $\check{\lambda}$ in Kleshchev's good lattice with respect to $\left(q^{\prime}, 1, \varepsilon^{\prime},\left(\varepsilon^{\prime}\right)^{2}\right.$, $\left.\cdots,\left(\varepsilon^{\prime}\right)^{m-1}\right)$, where $s:=n m / p$, then the sequence

$$
\begin{aligned}
& \underbrace{(\emptyset, \cdots, \emptyset)}_{p} \stackrel{r_{1}}{\rightarrow} \cdot \stackrel{m \ell+r_{1}}{\rightarrow} \ldots \stackrel{(p-m) \ell+r_{1}}{\rightarrow} \cdot \stackrel{r_{2}}{\rightarrow} \cdot \stackrel{m \ell+r_{2}}{\rightarrow} \ldots \stackrel{(p-m) \ell+r_{2}}{\rightarrow} . \\
& \ldots \stackrel{r_{s}}{\rightarrow} \stackrel{m \ell+r_{s}}{\rightarrow} \ldots \stackrel{(p-m) \ell+r_{s}}{\rightarrow} \lambda
\end{aligned}
$$

defines a path in Kleshchev's good lattice (w.r.t., $\left.\left(q, 1, \varepsilon, \varepsilon^{2}, \cdots, \varepsilon^{p-1}\right)\right)$ satisfying $\mathrm{h}^{m}(\lambda)=\lambda$.

Proof. This follows from (6.3), Theorem 3.6 and the realization of crystal graph as Kleshchev's good lattice.

We remark that one can derive a similar combinatorial result for FLOTW $p$ partitions by using the same arguments.

Theorem 6.10. Suppose that $K=\mathbb{C}, q, \varepsilon, q^{\prime}, \varepsilon^{\prime} \in \mathbb{C}$ be such that $\varepsilon=q^{\ell}$ (resp., $\left.\varepsilon^{\prime}=\left(q^{\prime}\right)^{\ell}\right)$ is a primitive $p$-th (resp., primitive $m$-th) root of unity, and $q$ (resp., $\left.q^{\prime}\right)$ is a primitive p $\ell$-th (resp., primitive $m \ell$-th) root of unity. Let $1 \leq m \leq$ $p$ be an integer such that $m \mid p$. Then $\widetilde{N}(m)$ is equal to the number of simple $\mathcal{H}_{q^{\prime}, 1, \varepsilon^{\prime}, \cdots,\left(\varepsilon^{\prime}\right)^{m-1}}(m, m n / p)$-modules, where $\mathcal{H}_{q^{\prime}, 1, \varepsilon^{\prime}, \cdots,\left(\varepsilon^{\prime}\right)^{m-1}}(m, m n / p)$ is the Ariki-Koike algebra with parameters $\left(q^{\prime}, 1, \varepsilon^{\prime}, \cdots,\left(\varepsilon^{\prime}\right)^{m-1}\right)$ and of size $m n / p$. In particular, for each integer $1 \leq \widetilde{m}<p$ such that $\widetilde{m} \mid p$, we get that

$$
\begin{aligned}
N(\widetilde{m}) & =\sum_{1 \leq m \leq \widetilde{m}, m \mid \widetilde{m}} \mu(\widetilde{m} / m) \widetilde{N}(m) \\
& =\sum_{1 \leq m \leq \widetilde{m}, m \mid \widetilde{m}} \mu(\widetilde{m} / m)\left(\# \operatorname{Irr}\left(\mathcal{H}_{q^{\prime}, 1, \varepsilon^{\prime}, \cdots,\left(\varepsilon^{\prime}\right)^{m-1}}(m, m n / p)\right)\right) .
\end{aligned}
$$

In the special case where $\ell=m=1$, the set $\check{\mathbb{B}}(\check{\Lambda})$ contains only one element-a highest weight vector, which corresponds to the empty partition $\emptyset$. Hence we have

Corollary 6.12. If $\ell=1$, then for any integer $n \geq 1$,

$$
\left\{\lambda \in K_{n} \mid \mathrm{h}(\lambda)=\lambda\right\}=\emptyset .
$$

Equivalently, $\widetilde{N}(1)=0=N(1)$. 
Corollary 6.13. With the same assumption as in Theorem [6.10, if $(p, n)=1$, then $\widetilde{N}(m)=0$ for any integer $1 \leq m<p$. In this case, for any irreducible $\mathcal{H}_{q}(p, n)$-module $D, D \downarrow_{\mathcal{H}_{q}(p, p, n)}$ remains irreducible.

Remark 6.14. Combing (6.2) with (6.11) we get an explicit formula for the number of simple $\mathcal{H}_{q}(p, p, n)$-modules in the case when $\varepsilon=q^{\ell}$ is a primitive $p$-th root of unity, and $q$ is a primitive $p \ell$-th root of unity. Note that (by Theorem 3.9) this formula is valid over any field $K$ as long as $K$ contains primitive $p$-th root of unity and $\mathcal{H}_{q}(p, p, n)$ is split over $K$. Our formula generalizes earlier results of Geck [19] on the Hecke algebra of type $D_{n}$ (i.e., of type $G(2,2, n)$ ). Note that Geck's method depends on explicit information on character tables and Kazhdan-Lusztig theory for Iwahori-Hecke algebras associated to finite Weyl group, which are not presently available in our general $G(p, p, n)$ cases.

We now deal with the remaining cases. That is, we assume the following setup: $K=\mathbb{C}, q, \varepsilon \in K . q$ is a primitive $d \ell$-th root of unity, $q^{\ell}=\varepsilon^{k}$ is a primitive $d$-th root of unity, and $1 \leq k<p$ is the smallest positive integer such that $\varepsilon^{k} \in\langle q\rangle$. In this case, we fix the order of parameters $\left\{1, \varepsilon, \cdots, \varepsilon^{p-1}\right\}$ as $\overrightarrow{\mathrm{Q}}:=\left(\overrightarrow{\mathrm{Q}}_{1}, \cdots, \overrightarrow{\mathrm{Q}}_{k}\right)$, where $\overrightarrow{\mathrm{Q}}_{i}=\left(\varepsilon^{i-1}, \varepsilon^{k+i-1}, \cdots, \varepsilon^{(d-1) k+i-1}\right)$ for $i=1,2, \cdots, k$. Let $\lambda \in K_{n}$. We write $\lambda=\left(\lambda^{[1]}, \cdots, \lambda^{[k]}\right)$ (concatenation of ordered tuples), where each $\lambda^{[i]}$ is a $d$-multipartition. By Theorem 3.8

$$
\mathrm{h}(\lambda)=\left(\mathrm{h}^{\prime}\left(\lambda^{[k]}\right), \lambda^{[1]}, \cdots, \lambda^{[k-1]}\right),
$$

where $\mathrm{h}^{\prime}$ is as defined in Corollary 3.7

As before, it suffices to derive a formula for $\widetilde{N}(m)$ for each integer $1 \leq m<p$ satisfying $m \mid p$. Suppose that $1 \leq a \leq \min \{m, k\}$ is the greatest common divisor of $m$ and $k$. By the division algorithm, we know that there exist integers $r_{1}, r_{2}$ such that $a=r_{1} k+r_{2} m$. Let $\lambda \in K_{n}$ be such that $\mathrm{h}^{m}(\lambda)=\lambda$. Then

$$
\begin{array}{r}
\left(\left(\mathrm{h}^{\prime}\right)^{r_{1}}\left(\lambda^{[1]}\right),\left(\mathrm{h}^{\prime}\right)^{r_{1}}\left(\lambda^{[2]}\right), \cdots,\left(\mathrm{h}^{\prime}\right)^{r_{1}}\left(\lambda^{[k]}\right)\right)=\mathrm{h}^{r_{1} k}(\lambda)=\mathrm{h}^{a-r_{2} m}(\lambda)=\mathrm{h}^{a}(\lambda) \\
=\left(\left(\mathrm{h}^{\prime}\right)\left(\lambda^{[k-a+1]}\right),\left(\mathrm{h}^{\prime}\right)\left(\lambda^{[k-a+2]}\right), \cdots,\left(\mathrm{h}^{\prime}\right)\left(\lambda^{[k]}\right), \lambda^{[1]}, \lambda^{[2]}, \cdots, \lambda^{[k-a]}\right) .
\end{array}
$$

It follows that

$$
\left(\mathrm{h}^{\prime}\right)^{r_{1}}\left(\lambda^{[i]}\right)= \begin{cases}\left(\mathrm{h}^{\prime}\right)\left(\lambda^{[k-a+i]}\right), & \text { if } 1 \leq i \leq a, \\ \lambda^{[i-a]}, & \text { if } a+1 \leq i \leq k .\end{cases}
$$

By an easy induction, we get that

$$
\left(\mathrm{h}^{\prime}\right)^{-r_{2} m / a}\left(\lambda^{[i]}\right)=\left(\mathrm{h}^{\prime}\right)^{r_{1} k / a-1}\left(\lambda^{[i]}\right)=\lambda^{[i]}, \forall 1 \leq i \leq k .
$$

We claim that there exist positive integers $r_{1}^{\prime}, r_{2}^{\prime}$ such that $a=r_{1}^{\prime} k+r_{2}^{\prime} m$ and $\left(r_{2}, r_{2}^{\prime}\right)=1$. In fact, since $a \mid m$, it is easy to check that $a=\left(-(m / a-1) r_{1}\right) k+$ $\left(-(m / a-1) r_{2}+1\right) m$. Taking $r_{1}^{\prime}=-(m / a-1) r_{1}, r_{2}^{\prime}=-(m / a-1) r_{2}+1$, we prove our claim.

Now applying our previous argument again, we get that

$$
\left(\mathrm{h}^{\prime}\right)^{-r_{2}^{\prime} m / a}\left(\lambda^{[i]}\right)=\lambda^{[i]}, \forall 1 \leq i \leq k .
$$

Let $x, y$ be two integers such that $x r_{2}+y r_{2}^{\prime}=1$. Then

$$
\left(\mathrm{h}^{\prime}\right)^{-m / a}\left(\lambda^{[i]}\right)=\left(\mathrm{h}^{\prime}\right)^{-x r_{2} m / a-y r_{2}^{\prime} m / a}\left(\lambda^{[i]}\right)=\lambda^{[i]}, \forall 1 \leq i \leq k .
$$


Equivalently, $\left(\mathrm{h}^{\prime}\right)^{m / a}\left(\lambda^{[i]}\right)=\lambda^{[i]}, \forall 1 \leq i \leq k$. We write $\lambda=\left(\lambda^{[1]}, \cdots, \lambda^{[k]}\right)$ (concatenation of ordered tuples), where each $\lambda^{[i]}$ is a $d$-multipartition. Let $n_{i}:=$ $\left|\lambda^{[i]}\right|$ for each $1 \leq i \leq k$. Then $\sum_{i=1}^{k} n_{i}=n$. Moreover, in this case we see (from (6.15)) that

$$
\lambda^{[k-j a+i]}=\left(\mathrm{h}^{\prime}\right)^{j r_{1}-1}\left(\lambda^{[i]}\right), \text { for } j=1,2, \cdots, k / a .
$$

As a consequence, $n_{1}+\cdots+n_{a}=n a / k$.

By Theorem 2.3, Lemma 2.4. Theorem 2.7 and our definition of the $p$-tuple $\overrightarrow{\mathrm{Q}}$, we know that $\lambda \in K_{n}$ if and only if for each $1 \leq i \leq k, \lambda^{[i]} \in \mathcal{K}_{n_{i}}$, where $\mathcal{K}_{n_{i}}$ denotes the set of Kleshchev $d$-multipartitions of $n_{i}$ with respect to $\left(q, 1, \varepsilon^{\prime}, \cdots, \varepsilon^{\prime d-1}\right)$, $\varepsilon^{\prime}=\varepsilon^{k}$. We define

$$
\begin{aligned}
& \widetilde{\Sigma}(k, m):=\left\{\begin{array}{l|l}
\left(\lambda^{[1]}, \cdots, \lambda^{[a]}\right) \vdash \frac{n a}{k} \mid \begin{array}{c}
\lambda^{[i]} \in \mathcal{K}_{n_{i}},\left(\mathrm{~h}^{\prime}\right)^{m / a}\left(\lambda^{[i]}\right)=\lambda^{[i]}, \\
\forall 1 \leq i \leq a, \sum_{i=1}^{a} n_{i}=\frac{n a}{k}
\end{array}
\end{array}\right\}, \\
& \widetilde{N}(k, m):=\# \widetilde{\Sigma}(k, m) .
\end{aligned}
$$

Lemma 6.16. With the above notations, the map which sends $\lambda=\left(\lambda^{[1]}, \cdots, \lambda^{[k]}\right)$ to $\bar{\lambda}:=\left(\lambda^{[1]}, \cdots, \lambda^{[a]}\right)$ defines a bijection from the set $\widetilde{\Sigma}(m)$ onto the set $\widetilde{\Sigma}(k, m)$.

Proof. Our previous discussion shows that the $p$-multipartition $\lambda=\left(\lambda^{[1]}, \lambda^{[2]}\right.$, $\left.\cdots, \lambda^{[k]}\right) \in \widetilde{\Sigma}(m)$ can be recovered from the $d a$-multipartition $\bar{\lambda}:=\left(\lambda^{[1]}, \lambda^{[2]}\right.$, $\left.\cdots, \lambda^{[a]}\right)$ and the automorphism $\mathrm{h}^{\prime}$. In particular, the above map is injective. It remains to prove the map is surjective.

Let $\alpha:=\left(\lambda^{[1]}, \cdots, \lambda^{[a]}\right) \in \widetilde{\Sigma}(k, m)$. Recall that $r_{1} k+r_{2} m=a$. For each integer $1 \leq i \leq a$, we define

$$
\lambda^{[k-j a+i]}:=\left(\mathrm{h}^{\prime}\right)^{j r_{1}-1}\left(\lambda^{[i]}\right) \text {, for } j=1,2, \cdots, k / a .
$$

This is well-defined, since $\left(\mathrm{h}^{\prime}\right)^{k r_{1} / a-1}\left(\lambda^{[i]}\right)=\left(\mathrm{h}^{\prime}\right)^{-r_{2} m / a}\left(\lambda^{[i]}\right)=\lambda^{[i]}$ (because $\alpha \in$ $\widetilde{\Sigma}(k, m))$. Note also that the above definition is equivalent to (6.15). Therefore, if we set $\lambda:=\left(\lambda^{[1]}, \cdots, \lambda^{[k]}\right)$, then the discussion above (6.15) implies that $\mathrm{h}^{r_{2} m}(\lambda)=\lambda$. Now recall that

$$
r_{1}^{\prime}=-(m / a-1) r_{1}, r_{2}^{\prime}=-(m / a-1) r_{2}+1, a=r_{1}^{\prime} k+r_{2}^{\prime} m .
$$

Therefore, for each integer $1 \leq i \leq a$, we have that

$$
\left(\mathrm{h}^{\prime}\right)^{j r_{1}^{\prime}-1}\left(\lambda^{[i]}\right)=\left(\mathrm{h}^{\prime}\right)^{-j(m / a-1) r_{1}-1}\left(\lambda^{[i]}\right)=\lambda^{[k-j a+i]}, \text { for } j=1,2, \cdots, k / a .
$$

Therefore, the discussion above (6.15) also implies that $\mathrm{h}^{r_{2}^{\prime} m}(\lambda)=\lambda$. Since $x r_{2}+$ $y r_{2}^{\prime}=1$, it follows that $\mathrm{h}^{m}(\lambda)=\mathrm{h}^{x r_{2} m+y r_{2}^{\prime} m}(\lambda)=\lambda$. In other words, $\lambda \in \widetilde{\Sigma}(m)$ with $\bar{\lambda}=\alpha$, as required. This proves the surjectivity, and hence completes the proof of the lemma.

Let $\widetilde{d}:=\left(d, \frac{m}{a}\right)$. Note that $\left(\mathrm{h}^{\prime}\right)^{d}=\mathrm{id}$. Therefore, $\left(\mathrm{h}^{\prime}\right)^{m / a}\left(\lambda^{[i]}\right)=\lambda^{[i]}$ if and only $\left(\mathrm{h}^{\prime}\right)^{\widetilde{d}}\left(\lambda^{[i]}\right)=\lambda^{[i]}$. Now applying Lemma 6.16, Theorem 6.10 and (6.3), we get

Theorem 6.17. Suppose that $K=\mathbb{C}, q, \varepsilon \in K . q$ is a primitive d $\ell$-th root of unity, $q^{\ell}=\varepsilon^{k}$ is a primitive $d$-th root of unity, and $1 \leq k<p$ is the smallest positive integer such that $\varepsilon^{k} \in\langle q\rangle$. Let $1 \leq m<p$ be an integer such that $m \mid p$. Let 


$$
\begin{aligned}
a=(m, k), \widetilde{d}:= & \left(d, \frac{m}{a}\right) . \text { Then } \\
& \widetilde{N}(m)=\widetilde{N}(k, m) \\
= & \sum_{n_{1}+\cdots+n_{a}=\frac{n a}{k}} \prod_{i=1}^{a}\left(\# \operatorname{Irr} \mathcal{H}_{q^{\prime \prime}, 1, \varepsilon^{\prime \prime}, \cdots,\left(\varepsilon^{\prime \prime}\right)^{\tilde{d}-1}}\left(\widetilde{d}, \frac{\widetilde{d} n_{i}}{d}\right)\right),
\end{aligned}
$$

where $\varepsilon^{\prime \prime}=\left(q^{\prime \prime}\right)^{\ell}$ is a primitive $\widetilde{d}$-th root of unity, and $q^{\prime \prime}$ is a primitive $(\widetilde{d} \ell)$-th root of unity. In particular, for each integer $1 \leq \widetilde{m}<p$ such that $\widetilde{m} \mid p$, we get that

$$
\begin{aligned}
& N(\widetilde{m})=\sum_{1 \leq m \leq \widetilde{m}, m \mid \widetilde{m}} \mu(\widetilde{m} / m) \widetilde{N}(m) \\
= & \sum_{1 \leq m \leq \widetilde{m}, m \mid \widetilde{m}} \mu(\widetilde{m} / m) \sum_{n_{1}+\cdots+n_{a}=\frac{n a}{k}} \prod_{i=1}^{a}\left(\# \operatorname{Irr} \mathcal{H}_{q^{\prime \prime}, 1, \varepsilon^{\prime \prime}, \cdots,\left(\varepsilon^{\prime \prime}\right)^{\tilde{d}-1}}\left(\widetilde{d}, \frac{\widetilde{d} n_{i}}{d}\right)\right) .
\end{aligned}
$$

Remark 6.19. Combing (6.2) with (6.18) we get an explicit formula for the number of simple $\mathcal{H}_{q}(p, p, n)$-modules in the case when $q$ is a primitive $d \ell$-th root of unity, $q^{\ell}=\varepsilon^{k}$ is a primitive $d$-th root of unity, and $1 \leq k<p$ is the smallest positive integer such that $\varepsilon^{k} \in\langle q\rangle$. Thus the problem on determining explicit formula for the number of simple $\mathcal{H}_{q}(p, p, n)$-modules is solved by our Theorem 6.10 and Theorem 6.17 in all cases. As before, this formula is valid over any field $K$ as long as $K$ contains primitive $p$-th root of unity and $\mathcal{H}_{q}(p, p, n)$ is split over $K$. Finally, we remark that, these explicit formulas strongly indicate that there are some new intimate connections between the representation of $\mathcal{H}_{q}(p, p, n)$ at roots of unity and the representation of various Ariki-Koike algebras of smaller sizes at various roots of unity. It seems very likely that the decomposition matrix of the latter can be naturally embedded as a submatrix of the decomposition matrix of the former. We will leave it to a future project.

\section{REFERENCES}

[1] S. Ariki, On the semi-simplicity of the Hecke algebra of $(Z / r Z)$ 乙 $\mathfrak{S}_{n}$, J. Alg. 169 (1994) 216-225. MR1296590 (95h:16020)

[2] S. Ariki, On the decomposition numbers of the Hecke algebra of $G(m, 1, n)$, J. Math. Kyoto Univ. 36 (1996) 789-808. MR1443748 (98h:20012)

[3] S. Ariki, Cyclotomic $q$-Schur algebras as quotients of quantum algebras, J. Reine Angew. Math. 513 (1999) 53-69. MR1713319(2001a:16065)

[4] S. Ariki, On the classification of simple modules for cyclotomic Hecke algebras of type $G(m, 1, n)$ and Kleshchev multi-partitions, Osaka J. Math. (4) 38 (2001) 827-837. MR1864465 (2002i:20004)

[5] S. Ariki, Representations of quantum algebras and combinatorics of Young tableaux, Translated from the 2000 Japanese edition and revised by the author, University Lecture Series, 26. American Mathematical Society, Providence, RI, 2002. MR1911030 (2004b:17022)

[6] S. Ariki and K. Koike, A Hecke algebra of $(\mathbb{Z} / r \mathbb{Z}) \imath \mathfrak{S}_{n}$ and construction of its representations, Adv. Math. 106 (1994) 216-243. MR1279219 (95h:20006)

[7] S. Ariki and A. Mathas, The number of simple modules of the Hecke algebras of type $G(r, 1, n)$, Math. Z. (3) 233 (2000) 601-623. MR1750939(2001e:20007)

[8] R.E. Borcherds, Generalized Kac-Moody algebras, J. Alg. (2) 115 (1988) 501-512. MR.943273 (89g:17004)

[9] J. Brundan, Modular branching rules and the Mullineux map for Hecke algebras of type $A$, Proc. London. Math. Soc. (3) 77 (1998) 551-581. MR1643413 (2000d:20007) 
[10] J. Brundan and A. Kleshchev, Hecke-Clifford superalgebras, crystals of type $A_{2 l}^{(2)}$ and modular branching rules for $\widehat{S}_{n}$, Representation Theory 5 (2001) 317-403. MR:1870595 (2002j:17024)

[11] M. Broué and G. Malle, Zyklotomische Heckealgebren, Astérisque 212 (1993) 119-189. MR.1235834 (94m:20095)

[12] C. W. Curtis and L. Reiner, Methods of Representations Theory, I, Wiley-Interscience, New York, 1981. MR0632548(82i:20001)

[13] R. Dipper and G. D. James, Representations of Hecke algebras of general linear groups, Proc. London. Math. Soc. (3) 52 (1986) 20-52. MR812444 (88b:20065)

[14] R. Dipper, G.D. James and A. Mathas, Cyclotomic $q$-Schur algebras, Math. Z. 229 (1998) 385-416. MR.1658581 (2000a:20033)

[15] R. Dipper and A. Mathas, Morita equivalence of Ariki-Koike algebras, Math. Z. 240 (2002) 579-610. MR1924022 (2003h:20011)

[16] O. Foda, B. Leclerc, M. Okado, J.-Y. Thibon and T. Welsh, Branching functions of $A_{(n-1)}^{(1)}$ and Jantzen-Seitz problem for Ariki-Koike algebras, Adv. Math. (2) 141 (1999) 322-365. MR:1671762 (2000f:17036)

[17] J. Fuchs, U. Ray and C. Schweigert, Some automorphisms of generalized Kac-Moody algebras, J. Alg. 191 (1997) 518-540. MR.1448807 (98g:17025)

[18] J. Fuchs, B. Schellekens and C. Schweigert, From Dynkin diagram symmetries to fixed point structures, Comm. Math. Phys. 180 (1996) 39-97. MR1403859 (98i:17021)

[19] M. Geck, On the representation theory of Iwahori-Hecke algebras of extended finite Weyl groups, Represent. Theory 4 (2000) 370-397. MR:1780716 (2002d:20006)

[20] G. Genet and N. Jacon, Modular representations of cyclotomic Hecke algebras of type $G(r, p, n)$, preprint, math.RT/0409297.

[21] J.J. Graham and G.I. Lehrer, Cellular algebras Invent. Math. 123 (1996) 1-34. MR.1376244 (97h:20016)

[22] T. Hayashi, $q$-analogues of Clifford and Weyl algebras-spinor and oscillator representations of quantum enveloping algebras, Comm. Math. Phys. 127 (1990) 129-144. MR1036118 (91a:17015)

[23] J. Hu, A Morita equivalence theorem for Hecke algebras of type $D_{n}$ when $n$ is even, Manuscr. Math. 108 (2002) 409-430. MR1923530 (2003h:20012)

[24] J. Hu, Crystal basis and simple modules for Hecke algebra of type $D_{n}$, J. Alg. (1) 267 (2003) 7-20. MR1993465 (2004d:20005)

[25] J. Hu, Modular representations of Hecke algebras of type $G(p, p, n)$, J. Alg. (2) 274 (2004) 446-490. MR:2043358 (2004m:20014)

[26] J. Hu, Branching rules for Hecke Algebras of Type $D_{n}$, Math. Nachr. 280 (2007) 93-104.

[27] J. Hu, The number of simple modules for the Hecke algebras of type $G(r, p, n)$, preprint, math.RT/0601572.

[28] N. Jacon, Représentations modulaires des algèbres de Hecke et des algèbres de Ariki-Koike, Ph.D. thesis, Université Claude Bernard Lyon I, 2004.

[29] N. Jacon, On the parameterization of the simple modules for Ariki-Koike algebras at roots of unity, J. Math. Kyoto Univ. 44 (2004) 729-767. MR2118039 (2006c:20008)

[30] M. Jimbo, K. Misra, T. Miwa and M. Okado, Combinatorics of representations of $U_{q}(\hat{s l}(n))$ at $q=0$, Comm. Math. Phys. 136 (1991) 543-566. MR1099695(93a:17015)

[31] M. Kashiwara, On crystal bases of the $q$-analogue of universal enveloping algebras, Duke J. Math. 63 (1991) 465-516. MR.1115118 (93b:17045)

[32] A. Kleshchev, Branching rules for the modular representations of symmetric groups III; some corollaries and a problem of Mullineux, J. London Math. Soc. 54 (1995) 25-38. MR 1395065 (96m:20019c)

[33] P. Littelmann, A Littlewood-Richardson rule for symmetrizable Kac-Moody algebras, Invent. Math. 116 (1994) 329-346. MR1253196 (95f:17023)

[34] P. Littelmann, Paths and root operators in representation theory, Ann. of Math. (2) 142 (1995) 499-525. MR.1356780 (96m:17011)

[35] A. Lascoux, B. Leclerc and J.-Y. Thibon, Hecke algebras at roots of unity and crystal bases quantum affine algebras, Comm. Math. Phys. 181 (1996) 205-263. MR1410572 (97k:17019)

[36] B. Leclerc and J.-Y. Thibon, Canonical bases of $q$-deformed Fock spaces, Int. Math. Res. Notices, 9 (1996) 447-456. MR1399410 (97h:17023) 
[37] G. Lusztig, Introduction to Quantum Groups, Progress in Math. 110 Birkhäuser, Boston, 1990. MR:1227098 (94m:17016)

[38] G. Malle and A. Mathas, Symmetric cyclotomic Hecke algebras, J. Alg. 205 (1998) 275-293. MR.1631350 (99g:20026)

[39] T. Misra and K.C. Miwa, Crystal bases for the basic representations of $U_{q}\left(\widehat{\mathfrak{s l}}_{n}\right)$, Comm. Math. Phys. 134 (1990) 79-88. MR1079801 (91j:17021)

[40] S. Naito and D. Sagaki, Lakshmibai-Seshadri paths fixed by a diagram automorphism, J. Alg. 245 (2001) 395-412. MR1868198(2002j:17026)

[41] S. Naito and D. Sagaki, Standard paths and standard monomials fixed by a diagram automorphism, J. Alg. 251 (2002) 461-474. MR.1900295 (2003d:17031)

[42] C. Pallikaros, Representations of Hecke algebras of type $D_{n}$, J. Alg. 169 (1994) 20-48. MR.1296580 (95k:20015)

[43] M. Varagnolo and E. Vasserot, On the decomposition matrices of the quantized Schur algebras, Duke Math. J. 100 (1999) 267-297. MR1722955 (2001c:17029)

[44] M. Vazirani, Irreducible modules over the affine Hecke algebra: a strong multiplicity one result, Ph.D. thesis, University of California at Berkeley, 1998.

Department of Applied Mathematics, Beijing Institute of Technology, Beijing, 100081, People's Republic of China

E-mail address: junhu303@yahoo.com.cn 